\title{
The WD40-Repeat Protein WDR-20 and the Deubiquitinating Enzyme USP-46 Promote Cell Surface Levels of Glutamate Receptors
}

\author{
Molly Hodul, ${ }^{1,2}$ Bethany J. Rennich, ${ }^{1,2}{ }^{\circledR}$ Eric S. Luth, ${ }^{1,3}$ Caroline L. Dahlberg, ${ }^{4}$ and ${ }^{\circledR}$ Peter Juo ${ }^{1}$ \\ ${ }^{1}$ Department of Developmental, Molecular and Chemical Biology, Tufts University School of Medicine, Boston, Massachusetts 02111, ${ }^{2}$ Program in \\ Neuroscience, Graduate School of Biomedical Sciences, Tufts University School of Medicine, Boston, Massachusetts 02111, ${ }^{3}$ Department of Biology, \\ Simmons University, Boston, Massachusetts 02115, and ${ }^{4}$ Department of Biology, Western Washington University, Bellingham, Washington 98225
}

Reversible modification of AMPA receptors (AMPARs) with ubiquitin regulates receptor levels at synapses and controls synaptic strength. The conserved deubiquitinating enzyme (DUB) ubiquitin-specific protease-46 (USP-46) removes ubiquitin from AMPARs and protects them from degradation in both Caenorhabditis elegans and mammals. Although DUBs are critical for diverse physiological processes, the mechanisms that regulate DUBs, especially in the nervous system, are not well understood. We and others previously showed that the WD40-repeat proteins WDR-48 and WDR-20 bind to and stimulate the catalytic activity of USP-46. Here, we identify an activity-dependent mechanism that regulates WDR-20 expression and show that WDR-20 works together with USP-46 and WDR-48 to promote surface levels of the C. elegans AMPAR GLR-1. usp-46, wdr-48, and wdr-20 loss-of-function mutants exhibit reduced levels of GLR-1 at the neuronal surface and corresponding defects in GLR-1-mediated behavior. Increased expression of WDR-20, but not WDR-48, is sufficient to increase GLR-1 surface levels in an usp-46-dependent manner. Loss of $u s p-46, w d r-48$, and $w d r-20$ function reduces the rate of local GLR-1 insertion in neurites, whereas overexpression of $w d r-20$ is sufficient to increase the rate of GLR-1 insertion. Genetic manipulations that chronically reduce or increase glutamate signaling result in reciprocal alterations in $w d r-20$ transcription and homeostatic compensatory changes in surface GLR-1 levels that are dependent on $w d r-20$. This study identifies $w d r-20$ as a novel activity-regulated gene that couples chronic changes in synaptic activity with increased local insertion and surface levels of GLR-1 via the DUB USP-46.

Key words: AMPA receptor; C. elegans; deubiquitinating enzyme; GLR-1; ubiquitin; USP46

\section{Significance Statement}

Deubiquitinating enzymes (DUBs) are critical regulators of synapse development and function; however, the regulatory mechanisms that control their various physiological functions are not well understood. This study identifies a novel role for the DUB ubiquitin-specific protease-46 (USP-46) and its associated regulatory protein WD40-repeat protein-20 (WDR-20) in regulating local insertion of glutamate receptors into the neuronal cell surface. This work also identifies WDR-20 as an activityregulated gene that couples chronic changes in synaptic activity with homeostatic compensatory increases in surface levels of GLR-1 via USP-46. Given that $35 \%$ of USP family DUBs associate with WDR proteins, understanding the mechanisms by which WDR proteins regulate USP-46 could have implications for a large number of DUBs in other cell types.

\section{Introduction}

Activity-dependent regulation of AMPA receptor (AMPAR) trafficking controls receptor levels at the synapse and is one of

Received May 5, 2020; revised Jan. 31, 2021; accepted Feb. 17, 2021.

Author contributions: M.H., B.J.R., E.S.L., C.L.D., and P.J. designed research; M.H., B.J.R., and E.S.L. performed research; C.L.D. contributed unpublished reagents/analytic tools; M.H., B.J.R., E.S.L., and P.J. analyzed data; M.H. and P.J. wrote the paper.

This work was supported by the National Science Foundation Grant IOS\#1353862 (to P.J.), the NIH Grant NS101534 (to P.J.), the NIH Postdoctoral Training Grant Fellowship 5K12GM074869 (to E.S.L.), the NIH Training Grant (Synapse Neurobiology) Fellowship T32NS061764 (to M.H.), and the NHH Predoctoral Training Grant Fellowship F31NS120586 (to B.J.R.). We thank the following for strains and reagents: Villu Maricq, Josh Kaplan, Itzhak Mano, the Caenorhabditis Genetics Center [funded by the National Institutes of Health (NIH) Office of Research Infrastructure Programs Grant P40 the major mechanisms underlying both Hebbian and homeostatic plasticity (Huganir and Nicoll, 2013). Several trafficking pathways regulate the number of AMPARs at synapses including motor-dependent transport, insertion into the plasma

0D010440], the C. elegans knock-out consortium, and Shohei Mitani from the National Bioresource. We also thank Victor Hatini for assistance with the confocal microscope.

M. Hodul's present address: Department of Neurology, University of California, San Francisco, California 94158.

The authors declare no competing financial interests.

Correspondence should be addressed to Peter Juo at peter.juo@tufts.edu.

https://doi.org/10.1523/JNEUROSCI.1074-20.2021

Copyright $\odot 2021$ the authors 
membrane, lateral diffusion to and from extrasynaptic sites, and endocytosis into internal compartments (Anggono and Huganir, 2012). After internalization, AMPARs are sorted in endosomes and are either targeted for lysosomal degradation or recycled back to the neuronal cell surface (Ehlers, 2000; Lee et al., 2004). Ubiquitination is a posttranslational modification that is widely used to target membrane proteins for degradation (Lauwers et al., 2010). Ubiquitin has emerged as a critical regulator of synapse development and function (Bingol and Sheng, 2011), that can control glutamatergic signaling by directly regulating AMPAR trafficking and degradation (Widagdo et al., 2017). Studies in Caenorhabditis elegans first revealed that AMPAR trafficking was regulated by ubiquitin. Direct conjugation of ubiquitin to the $C$. elegans AMPAR GLR-1 promotes receptor internalization and degradation in lysosomes (Burbea et al., 2002; Chun et al., 2008). Subsequent studies using cultured rodent neurons showed that acute or chronic AMPAR activation results in ubiquitination and degradation of all four AMPAR receptor subunits (Schwarz et al., 2010; Fu et al., 2011; Hou et al., 2011; Lin et al., 2011; Lussier et al., 2011; Widagdo et al., 2015).

Several ubiquitin ligases can either directly or indirectly regulate invertebrate (Juo and Kaplan, 2004; van Roessel et al., 2004; Dreier et al., 2005; Schaefer and Rongo, 2006; Park et al., 2009) and vertebrate (Schwarz et al., 2010; Fu et al., 2011; Lussier et al., 2012; Lin and Man, 2014) AMPAR trafficking. However, ubiquitination is a reversible modification, and much less is known about the deubiquitinating enzymes (DUBs) that proteolytically remove ubiquitin from substrates. The human genome encodes for 500-600 ubiquitin ligases but only $\sim 100$ DUBs (Mevissen and Komander, 2017). Despite the lower number of DUB genes, recent studies indicate that many DUBs regulate specific substrates and cellular processes, suggesting that DUB function is tightly regulated in vivo (Clague et al., 2019). However, the molecular mechanisms that regulate DUBs, particularly in response to changes in neuronal activity, are not well understood.

We previously identified ubiquitin-specific protease-46 (USP46) as the first DUB to regulate glutamate receptors. We found that USP-46 removes ubiquitin from GLR-1 and protects it from degradation in the lysosome in C. elegans (Kowalski et al., 2011). Similarly, mammalian USP46, which colocalizes with AMPARs and PSD95 at synapses in cultured rodent neurons, deubiquitinates and stabilizes GluA1 and GluA2 (Huo et al., 2015). Lossof-function of usp-46 in C. elegans (Kowalski et al., 2011; Dahlberg and Juo, 2014) or rodent neurons (Huo et al., 2015) leads to reduced surface levels of AMPARs and decreased synaptic function. Mammalian USP46, and its close homolog USP12, have low intrinsic catalytic activity. However, binding of USP46 or USP12 to the WD40-repeat proteins, WDR48 (also known as UAF1) and WDR20, stimulates their catalytic activity, with WDR20 further enhancing the activity of USP46/WDR48 complexes (Cohn et al., 2009; Sowa et al., 2009; Kee et al., 2010; Faesen et al., 2011).

We previously found that the C. elegans homologs, WDR-48 and WDR-20, also bind to and stimulate the catalytic activity of USP-46 (Dahlberg and Juo, 2014). Overexpression of $w d r-48$ and $w d r-20$ resulted in deubiquitination of GLR-1 and increased total levels of the receptor in vivo, as well as increased surface GLR-1 levels in cultured C. elegans embryonic neurons. However, the effect of loss-of-function $w d r$ mutants and the precise mechanism by which the USP-46/WDR complex regulates GLR-1 surface levels are unknown.

Here, we use transgenic animals expressing GLR-1 tagged with $\mathrm{pH}$-sensitive Superecliptic pHluorin (SEP) to quantitatively examine the relative contributions and mechanism by which the WDR proteins and USP-46 regulate GLR-1 surface levels in vivo. We find that USP-46, WDR-48, and WDR-20 localize to Syntaxin-13-labeled endosomes and that WDR-20 regulates surface levels of GLR-1 by promoting local insertion. Additionally, chronic changes in glutamate signaling regulates $w d r-20$ transcription and surface GLR-1 levels. This study identifies $w d r-20$ as a novel activity-dependent gene that couples chronic changes in synaptic activity with surface levels of GLR-1 via the DUB USP-46.

\section{Materials and Methods}

Strains

The following strains were used for experiments described in this manuscript:

N2 (Bristol) wild-type, akIs201 (Prig-3::SEP::mCherry::GLR-1) V (gift from Villu Maricq), pzIs45 (Prig-3::SEP:::mCherry::GLR-1), usp-46 (ok2232) III, $w d r-48$ (tm4575) III, $w d r-20$ ( $g k 547140) \mathrm{V}, p z E x 230$ (Pglr-1::WDR-20), pzEx231 (Pglr-1::WDR-48), pzIs25 (Pglr-1::WDR-20; Pglr-1::WDR-48) I, pzEx224 (Pglr-1::USP-46), eat-4 (n2474) III, pzIs38 (Pwdr-20::NLS-GFPLacZ) III, pzEx463 (Pnmr-1::dsRed), glt-3 (bz34) glt-6 (tm1316) glt-7 (tm1641) IV (gift from Itzhak Mano), pzEx93 (Pglr-1::mCherry::USP-46), pzEx274 (Pglr-1::mCherry::WDR-48), pzEx264 (Pglr-1::mCherry::WDR-20), pzEx135 (Pglr-1::venus::RAB-5), nuIs232 (Pglr-1::GFP-Syntaxin-13; gift from Josh Kaplan). All strains were maintained at $20^{\circ} \mathrm{C}$ as described previously (Brenner, 1974).

The usp-46 (ok2232) allele contains a 480 amino acid deletion that removes the majority of the protein product, including the critical histidine and aspartic acid residues of the catalytic triad and thus is a functional null (Kowalski et al., 2011). The $w d r-48$ (tm4575) allele contains a 132 -amino acid deletion that disrupts WD40-repeat domains 5-7, and results in the introduction of a premature stop codon downstream of the deletion (Dahlberg and Juo, 2014). The $w d r-20$ ( $g k 547140)$ allele contains a non-sense mutation that results in a premature stop codon at amino acid 288 in the WDR-20 protein coding sequence (Dahlberg and Juo, 2014).

Constructs, transgenes, and germ-line transformation

akIs201 was a gift from Villu Maricq (Hoerndli et al., 2015). pzIs45 was created by injecting DM\#1983 (Prig-3::SEP::-mCherry::GLR-1; gift from Villu Maricq) at $50 \mathrm{ng} / \mu \mathrm{l}$ into unc-119 (ed3) animals with the coinjection plasmid pCFJ\#350 which contains an $u n c-119$ rescuing transgene $(50 \mathrm{ng} / \mu \mathrm{l})$ to create pzEx460 followed by UV integration using a UV Stratalinker. pzIs45 was backcrossed three times before imaging. pzEx224, pzEx230, $p z E x 231$, and pzIs25 were previously described (Dahlberg and Juo, 2014).

pzIs38 was created by injecting plasmid FJ\#101 (Pwdr-20::NLS-GFPLacZ; $50 \mathrm{ng} / \mu \mathrm{l})$ with the coinjection marker Pttx-3::dsRed $(50 \mathrm{ng} / \mu \mathrm{l})$ to create pzEx230 (Dahlberg and Juo, 2014) followed by UV integration using a UV Stratalinker. pzIs 38 was backcrossed 5 times before imaging. FJ\#138 (Pnmr-1::dsRed) was generated by cloning Pnmr-1 ( 1 kb) from FJ\#129 (Pnmr-1::USP-46::GFP) into the KP\#889 vector expressing dsRed using SphI and BamHI sites. pzEx463 was created by injecting plasmid FJ\#138 (10 ng/ $\mu$ l) into unc-119 (ed3) animals with coinjection plasmid pCFJ\#350 which contains an unc-119 rescuing transgene (50 ng/ $\mu \mathrm{l})$ and pBluescript (40 ng/ $\mu \mathrm{l})$. pzEx93 (Pglr-1::mCherry::USP-46;Pmyo2::NLS-mCherry) and pzEx135 (Pglr-1::venus::Rab-5; Pttx-3::GFP) were previously described (Kowalski et al., 2011). pzEx274 was created by injecting the plasmid FJ\#103 (Pglr-1::mCherry::WDR-48) with the coinjection marker Pttx-3::dsRed. FJ\#103 was generated by subcloning mCherry in-frame after the start codon of the $w d r-48$ cDNA using a Not I site introduced via PCR in FJ\#98 (Pglr-1::WDR-48) to create WDR-48 tagged at the $\mathrm{N}$ terminus with mCherry. pzEx264 was created by injecting plasmid FJ\#102 (Pglr-1::mCherry::WDR-20) with the coinjection marker Pmyo-2::NLS-GFP. FJ\#102 was generated by subcloning mCherry in-frame after the start codon of the $w d r-20$ cDNA (long-splice form) using a NotI site introduced via PCR in FJ\#97 (Pglr-1::WDR-20) to create WDR-20 tagged at the $\mathrm{N}$ terminus with mCherry. nuIs232 (Pglr-1::GFP-Syntaxin-13; gift from Joshua Kaplan) was previously described (Chun et al., 2008). 
GLR-1 fluorescence imaging

Fluorescence imaging of SEP::mCherry::GLR-1 (akIs201 or pzIs45) was performed as follows. Images were collected on a Carl Zeiss LSM800 Confocal microscope with a $63 \times$ Plan Apochromat (1.4 numerical aperture) objective using Zen Blue software (Zeiss). Larval-stage 4 (L4) animals were immobilized in a droplet of M9 containing $2.3 \mathrm{~mm}$ Levamisole (Tetramisole, Sigma) and placed on a $2 \%$ agarose pad containing Levamisole. For the ventral nerve cord (VNC), images were acquired with an acquisition area of $100 \times 60 \mu \mathrm{m}$ just posterior to the RIG neuronal cell bodies in $7-\mu \mathrm{m}$ Z-series stacks. Images were acquired with the 488 -nm laser set to $8.94 \%$ power and $776-\mathrm{V}$ gain, and the 561 $\mathrm{nm}$ laser set to $5.19 \%$ power and $867-\mathrm{V}$ gain. For the nerve ring (NR), images were acquired with an acquisition area of $50 \times 50 \mu \mathrm{m}$ centered on the NR in $10-\mu \mathrm{m}$ Z-series stacks which encompassed the entire NR. Images were acquired with the $488-\mathrm{nm}$ laser set to $8.94 \%$ power and $776-\mathrm{V}$ gain, and the $561-\mathrm{nm}$ laser set to $5.19 \%$ power and $867-\mathrm{V}$ gain. Maximum intensity projections of Z-series stacks were made after applying a single pixel filter to reduce noise before quantitation.

To quantify SEP and mCherry fluorescence in VNC puncta, the background threshold was first defined with a minimum threshold of 70 and a maximum threshold of 255 using Image (NIH). An identical region of interest (ROI) was drawn around an anterior region of the VNC in both the SEP and mCherry images for the same animal and the integrated density (product of area and mean gray intensity) of the thresholded VNC puncta were measured. To quantify SEP and mCherry fluorescence in NR puncta, the background threshold was first defined with a minimum threshold of 60 and a maximum threshold of 255 using Image $(\mathrm{NIH})$. The NR was straightened by drawing a line along the NR and using the Straighten tool in ImageJ. An identical ROI was used for the straightened NR in both the SEP and mCherry images for the same animal and the integrated density (product of area and mean gray intensity) of the thresholded NR puncta were measured. Following quantification, orthogonal projections were globally processed in Photoshop (Adobe) to further reduce noise and increase contrast using identical settings for all images. All values were normalized to the average intensity measured for wild-type before graphing.

Fluorescence recovery after photobleaching (FRAP)

A Zeiss LSM800 confocal microscope, described above, was used for SEP::mCherry::GLR-1 FRAP experiments. Prebleach and postbleach images were acquired using an image acquisition area of $100 \times 60 \mu \mathrm{m}$ with the 488 -nm laser set to $5.8 \%$ power and $776-\mathrm{V}$ gain, and the $561-$ $\mathrm{nm}$ laser set to $3.6 \%$ power and $867-\mathrm{V}$ gain. A prebleach Z-series image stack was obtained with a $7-\mu \mathrm{m}$ total depth. Immediately after, photobleaching was performed using an acquisition area of $50 \times 30 \mu \mathrm{m}$, which is half the size of the image area, with the $488-\mathrm{nm}$ laser set to $30 \%$ power and the $561-\mathrm{nm}$ laser set to $10 \%$ power. A postbleach image was taken immediately after bleaching using the original imaging settings, followed by manual image acquisition at $2.5,5$, and 10 min postbleach. Maximum intensity projections of Z-series stacks were used for quantitative analyses of fluorescent puncta. A single pixel filter was applied to reduce noise.

To quantify SEP and mCherry fluorescence in VNC puncta, the background threshold was first defined with a minimum threshold of 25 and a maximum threshold of 255 using Image (NIH). An ROI was drawn around the bleached portion of the image, which was used for all images in the dataset, and the integrated density (product of area and mean gray intensity) of thresholded VNC puncta was measured. Following quantification, orthogonal projections were globally processed in Photoshop 8 (Adobe) to further reduce noise and increase contrast using identical settings for all images. All values were normalized to their respective prebleach intensity before graphing.

\section{Endosome colocalization imaging}

A Zeiss LSM800 confocal microscope, described above, was used to capture images of mCherry tagged USP-46, WDR-48, and WDR-20 with GFP::Syntaxin-13 or Venus::RAB-5. L4 worms were immobilized as above. All images were acquired with an acquisition area of $50.71 \times$ $50.71 \mu \mathrm{m}$ centered just posterior to the NR where the cell bodies of many GLR-1-expressing neurons reside, including the AVA cell body. A 14.7- $\mu \mathrm{m}$ Z-series stack was used to capture the AVA cell body. For the Venus::RAB-5 images, the 488 -nm laser was set to $0.99 \%$ power with $535-\mathrm{V}$ gain and the $561-\mathrm{nm}$ laser was set to $0.99 \%$ power with $715-\mathrm{V}$ gain. For the GFP::Syntaxin-13 images, the 488-nm laser was set to $1.00 \%$ power with $550-\mathrm{V}$ gain, and the $561-\mathrm{nm}$ laser was set to $0.98-$ $0.99 \%$ power with $650-$ to $750-\mathrm{V}$ gain. A single representative plane from each Z-series stack was chosen for display and individually processed using ImageJ to reduce noise and increase contrast and then cropped using Adobe Illustrator.

\section{Behavioral assays}

All behavioral assays were performed using at least 20 young adult hermaphrodites over at least 3 independent experiments and by an experimenter blinded to the genotypes of the animals being tested. Nose-touch assays were performed as described previously (Kaplan and Horvitz, 1993). Briefly, worms were placed on 60-mm NGM agar plates containing a thin lawn of OP50 Escherichia coli spotted the day before the experiment and then assayed for responsiveness to light touch on the nose as determined by perpendicular contact with a human eyelash during forward locomotion. Ten trials were performed for each animal, and backward movement greater than the distance from the nose to the terminal bulb of the pharynx was counted as a reversal.

\section{Experimental design and statistical analysis}

All experiments were performed using L4 larval stage hermaphrodite $C$. elegans as determined by the canonical vulval shape at this stage. All mutant strains were back-crossed at least two times with N2 wild-type animals. The total number of animals measured for each condition is indicated in the figure legends. All experiments were performed at least three times.

All statistical analyses were performed using GraphPad Prism 8 software (GraphPad). For comparisons of two groups, unpaired Student's $t$ test was used. Statistical values for unpaired $t$ test were provided as $(p, t$, df) in the figure legends. For comparisons of three or more groups, oneway ANOVA followed by Tukey's multiple comparison tests were used. For comparisons of multiple groups at different time points, two-way ANOVA followed by Tukey's multiple comparison tests were used. Statistical values for ANOVAs were provided as $\left(F_{(\mathrm{DFn}, \mathrm{DFd})}, p\right)$ in the figure legends. The level of significance was set as $p<0.05$. Values that differ significantly from wild-type are indicated in the figures as follows: $\# p \leq 0.05, * p \leq 0.01, * * p \leq 0.001$. All graphs show mean \pm SEM.

\section{Results}

\section{USP-46 and co-factors WDR-48 and WDR-20 promote surface levels of GLR-1}

The DUB USP-46 regulates total protein levels of GLR-1 glutamate receptors in the ventral nerve cord (VNC) of C. elegans by deubiquitinating GLR-1 and protecting it from degradation in the lysosome (Kowalski et al., 2011). usp-46 loss-of-function mutants exhibit strong defects in GLR-1-mediated behaviors and have reduced levels of HA::GLR-1 (epitope-tagged on its extracellular domain) at the cell surface based on injection of fluorescent anti-HA antibodies into the pseudocoelom (Kowalski et al., 2011). Interestingly, we previously noticed that loss of usp- 46 has stronger effects on both GLR-1 surface levels and GLR-1-mediated behaviors than on total GLR-1 levels, suggesting that reduced degradation is not the only mechanism by which USP-46 controls cell surface GLR-1 levels. This observation prompted us to further investigate the mechanism by which USP-46 regulates GLR-1 surface levels. We first sought to confirm our previous observations using a more direct and quantitative in vivo method to monitor surface levels of GLR-1. We used a previously-described transgenic strain (akIs201) (Hoerndli et al., 2013) that expresses a functional GLR-1 tagged on its extracellular domain with both the $\mathrm{pH}$-sensitive fluorophore SEP (Miesenböck et al., 1998) and mCherry (SEP:: 
SEP::mCherry::GLR-1 in VNC

A

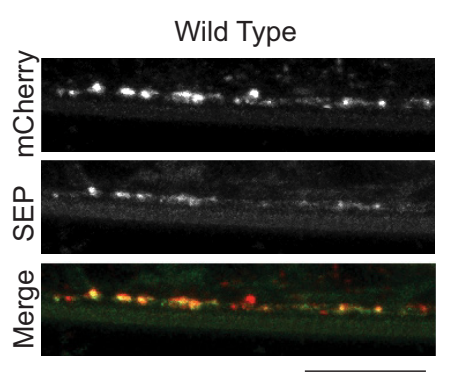

$10 \mu \mathrm{M}$

B

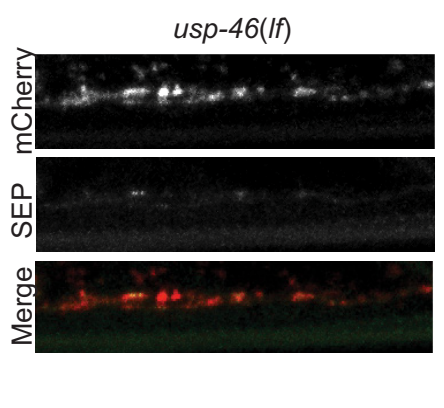

D

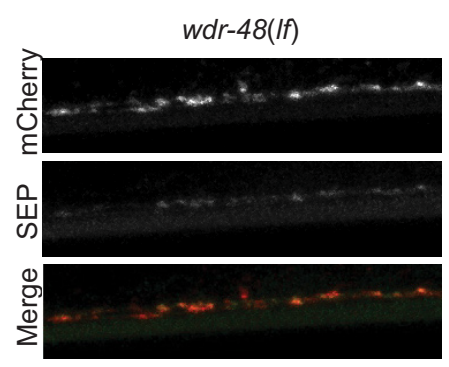

$\mathbf{F}$

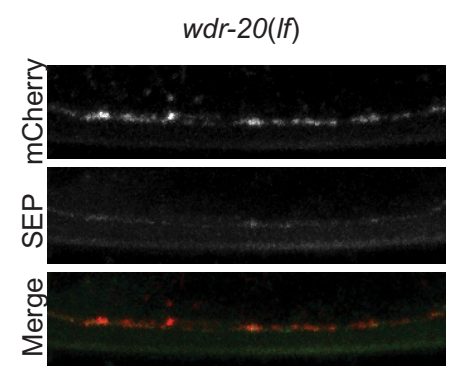

SEP::mCherry::GLR-1 in VNC
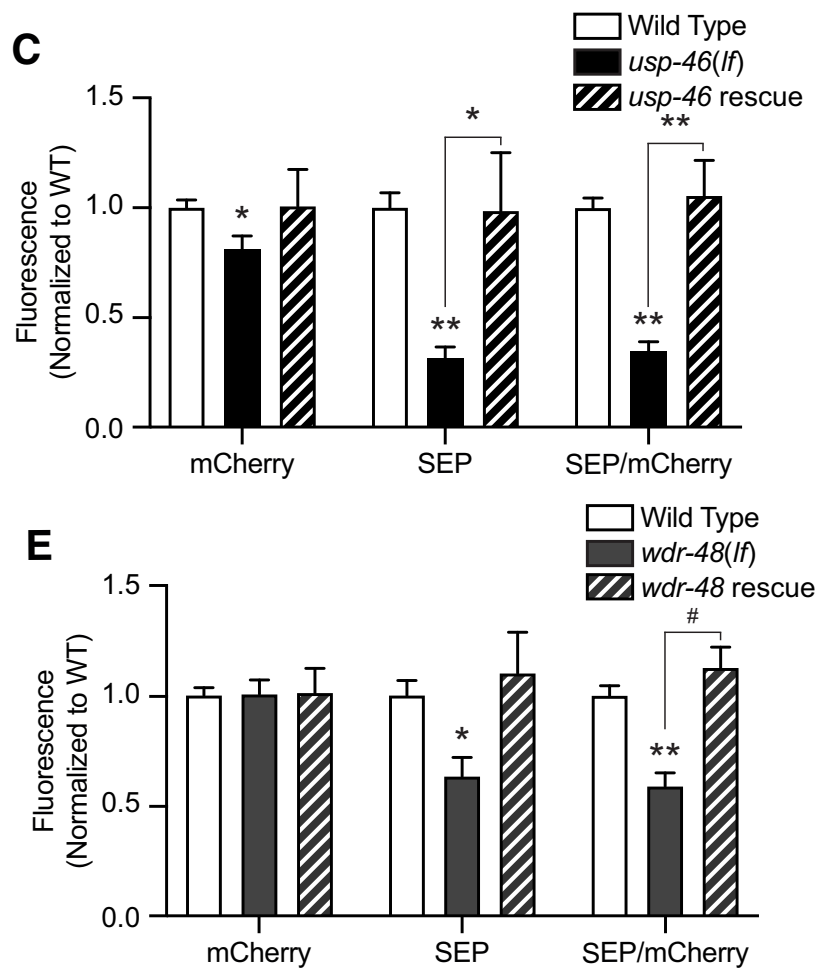

G

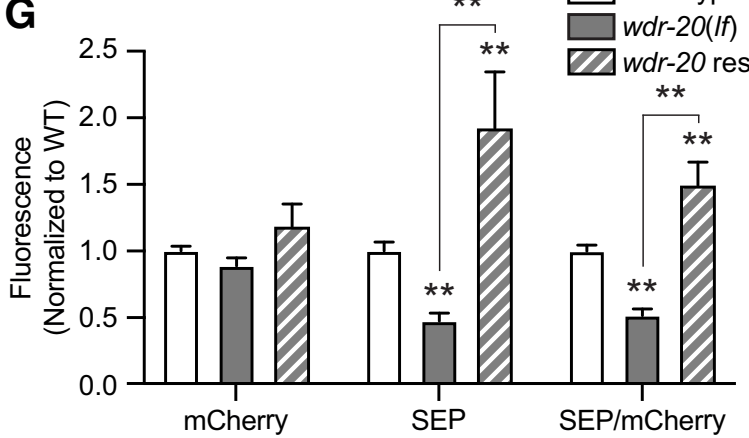

Figure 1. Surface levels of GLR-1 are regulated by USP-46 and co-factors WDR-48 and WDR-20. $A$, Representative images of SEP:::mCherry::GLR-1 in the VNCS of L4 stage larval animals harboring a SEP::.mCherry::GLR-1 integrated transgene (akls201). B, Representative images of SEP::.mCherry::GLR-1 in a usp-46 (ok2232) mutant background. C, Quantification of the fluorescence intensity of mCherry (total GLR-1), SEP (surface GLR-1), and the ratio of SEP/mCherry (surface to total GLR-1, normalized to wild type) for animals expressing akls201 in either wild-type $(n=122)$, usp-46 (ok2232) ( $n=69)$, or usp-46 (ok2232) mutants expressing wild-type usp-46 CDNA under control of the glr- 1 promoter [usp-46 rescue (pzEx224 line 1), $n=15$ ] are shown. One-way ANOVA revealed a significant effect of genotype for mCherry $\left(F_{(2,243)}=3.861, p=0.0224\right)$, $\operatorname{SEP}\left(F_{(2,243)}=19.37, p<0.0001\right)$, and $\operatorname{SEP} / \mathrm{mCherry}\left(F_{(2,243)}=39.36, p<0.0001\right)$. $\boldsymbol{D}$, Representative images of SEP:::mCherry::GLR-1 in a wdr-48 (tm4575). E, Quantification of mCherry intensity, SEP fluorescence intensity, and the ratio of SEP/mCherry (normalized to wild type) for animals expressing akls201 in either wild-type $(n=122)$, wdr-48 (tm4575) $(n=49)$ mutants or wdr-48 (tm4575) mutants expressing wild-type wdr-48 cDNA under control of the glr-1 promoter [wdr-48 rescue (pzEx231 line 1), $n=9$ ]. One-way ANOVA revealed no significant effect of genotype for mCherry $\left(F_{(2,216)}=0.0051, p=0.9949\right)$, but a significant effect of genotype for $\operatorname{SEP}\left(F_{(2,216)}=4.117, p=0.0176\right)$ and SEP/mCherry $\left(F_{(2,216)}=11.79, p<0.0001\right)$. $\boldsymbol{F}$, Representative images of SEP:::mCherry::GLR-1 in wdr-20 ( $\left.g k 547140\right)$ mutants. $\boldsymbol{G}$, Quantification of the fluorescence intensity of mCherry (total GLR-1), SEP (surface GLR-1), and the ratio of SEP/mCherry (surface to total GLR-1, normalized to wild type) for L4 stage larval animals harboring a SEP:: mCherry::GLR-1 integrated transgene (pzls45) in either wild-type $(n=40)$, wdr-20 (gk547140) $(n=51)$ mutants, or $w d r-20$ ( $g k 547140)$ mutants expressing wild-type wdr-20 CDNA expressed under control of the glr- 1 promoter [wdr-20 rescue ( $p z E x 230$ line 1), $n=12$ ]. One-way ANOVA revealed no significant effect of genotype for $m C h e r r y ~\left(F_{(2,222)}=2.418, p=0.0915\right)$, but a significant effect of genotype for $\operatorname{SEP}\left(F_{(2,222)}=16.99, p<0.0001\right)$ and SEP/mCherry $\left(F_{(2,222)}=23.62, p<0.0001\right)$. All graphs show mean \pm SEM. Values that differ significantly from the wildtype (ANOVA, Tukey's multiple comparison test) are indicated as follows: $\# p \leq 0.05, * p \leq 0.01, * * p \leq 0.001$.

mCherry::GLR-1) in GLR-1-expressing AVA interneurons. GLR-1 tagged with fluorescent proteins, such as GFP (Rongo et al., 1998) or SEP::mCherry (Hoerndli et al., 2013), localizes to puncta in the nerve ring (NR) and along the VNC, the vast majority of which are thought to represent postsynaptic sites (Rongo et al., 1998; Burbea et al., 2002). SEP fluorescence can be used to estimate the levels of GLR-1 at the cell surface, because it fluoresces in the neutral $\mathrm{pH}$ of the extracellular space but is quenched in the acidic endosomal environment. In contrast, mCherry fluoresces at both the cell surface and in internal compartments and thus represents an estimate of total GLR-1 levels. A previous study showed that SEP::GLR-1 signal in the VNC could be quenched by an extracellular acid wash confirming that SEP fluorescence represents receptors at the cell surface (Wang et al., 2012). Additionally, we found that SEP fluorescence increases dramatically (Luth et al., 2021) when GLR-1 endocytosis is blocked by mutation of the clathrin adaptin unc-11/ AP180 (Burbea et al., 2002). 
Confocal imaging of SEP::mCherry::GLR-1 levels revealed that usp-46 (ok2232) loss-of-function mutants (for allele information, see Materials and Methods) exhibit a $\sim 20 \%$ reduction in mCherry fluorescence (total GLR-1, $p=0.0184$ ) and a $\sim 70 \%$ reduction in SEP fluorescence (surface GLR-1, $p<0.0001$ ) in the VNC (Fig. $1 A-C$ ). Analysis of the SEP/mCherry fluorescence ratio confirms that usp-46 mutants have a larger reduction in surface versus total GLR-1 levels, consistent with previous observations based on anti-HA antibody labeling (Kowalski et al., 2011). These results provide further evidence for our hypothesis that in addition to protecting GLR-1 from degradation (Kowalski et al., 2011), USP-46 has another major function, which is to promote surface levels of GLR-1. We found that expression of wild-type usp-46 cDNA in GLR-1-expressing neurons (using the $g l r-1$ promoter) rescues the reductions in SEP and mCherry fluorescence observed in $u s p-46$ mutants, indicating that USP-46 functions in GLR-1-expressing neurons to regulate GLR-1 (Fig. 1C).

The WD40-repeat proteins WDR-48 and WDR-20 bind and activate the catalytic activity of USP-46 and its homologs from yeast to mammals, with WDR-20 interaction synergistically enhancing the activity of the USP-46/WDR-48 complex (Cohn et al., 2009; Sowa et al., 2009; Kee et al., 2010; Kouranti et al., 2010; Faesen et al., 2011; Dahlberg and Juo, 2014; Hodul et al., 2017). We previously showed that overexpression of $w d r-48$ and $w d r-20$ in vivo increases total GLR-1 levels and a GLR-1-mediated locomotion reversal behavior (Dahlberg and Juo, 2014). In addition, based on anti-HA antibody staining under non-permeabilized conditions, overexpression of both $w d r-48$ and $w d r-20$ together increased surface levels of HA::GLR-1 in cultured embryonic neurons from C. elegans (Dahlberg and Juo, 2014). However, the relative contributions of $w d r-48$ and $w d r-20$ or their effects in vivo on surface GLR-1 levels are not known. Here, we investigated whether loss of $w d r-48$ or $w d r-20$ alters GLR-1 surface levels in vivo. Similar to usp-46 mutants, we found that $w d r-48$ ( $t m 4575)$ and $w d r-20$ ( $g k 547140)$ loss-offunction mutants (for allele information, see Materials and Methods) have decreased SEP fluorescence (surface GLR-1; WT vs $w d r-48, p=0.0147$; WT vs $w d r-20, p=0.0003)$ and SEP/ mCherry ratios (WT vs $w d r-48, p<0.0001$; WT vs $w d r-20$, $p<0.0001$; Fig. $1 D-G$ ). Expression of wild-type $w d r-48$ or $w d r-$ $20 \mathrm{cDNA}$ in GLR-1-expressing neurons (using the $g l r-1$ promoter) rescues the GLR-1 defects observed in their respective mutant backgrounds (Fig. 1E,G). The lack of an effect of $w d r-$ 48 or $w d r-20$ mutants versus $u s p-46$ on mCherry fluorescence (total GLR-1) suggest that low levels of USP-46 catalytic activity in the absence of WDR-48 or WDR-20 may be sufficient to protect GLR-1 from degradation. Alternatively, other unidentified factors may regulate USP-46 activity to control degradation. Taken together, these data indicate that USP-46 and the WDR proteins act in GLR-1-expressing neurons to promote surface levels of GLR-1 in vivo.

\section{Overexpressed WDR-20 but not USP-46 or WDR-48 increases GLR-1 surface abundance}

We next tested whether overexpression of $u s p-46$ in a wild-type background would increase surface levels of GLR-1. We found that overexpression of $u s p-46$ alone in GLR-1-expressing neurons $[u s p-46(x s)]$ is not sufficient to increase SEP fluorescence (surface GLR-1; Fig. 2A). Interestingly, we found that overexpression of $w d r-48$ and $w d r-20$ in GLR-1-expressing neurons $[w d r-48(x s) ; w d r-20(x s)]$ is sufficient to increase SEP fluorescence (surface GLR-1, $p<0.0001$ ) by almost twofold (Fig. $2 B$ ). This effect was blocked in $u s p-46$ mutants, suggesting that the ability of WDR-48 and WDR-20 to increase surface GLR-1 levels is dependent on endogenous usp-46. We next determined the contribution of each WDR protein individually to the regulation of surface GLR-1 levels. We found that overexpression of $w d r-48$ alone $[w d r-48(x s)]$ has no effect on SEP fluorescence (surface GLR-1; Fig. 2C). In contrast, overexpression of $w d r-20$ alone $[w d r-20(x s)]$ was sufficient to increase SEP fluorescence (surface GLR-1, $p=0.0109$; Fig. 2D). Because WDR-48 can enhance binding of WDR-20 to USP-46 (Kee et al., 2010), we tested whether the effect of $w d r-20$ is dependent on $w d r-48$. We found that the increase in SEP fluorescence (surface GLR-1) observed in $w d r-20$ $(x s)$ animals is blocked in $w d r-48$ loss-of-function mutants (Fig. $2 D)$, indicating that endogenous $w d r-48$ is required for the effect of $w d r-20$ on GLR-1. Collectively, these data show that increased expression of $w d r-20$, but not $w d r-48$, is sufficient to promote surface levels of GLR-1. Our findings also suggest that $w d r-20$ expression may be limiting in vivo and that regulating $w d r-20$ expression might be an effective mechanism to control surface levels of GLR-1. Together with previous studies, our data are consistent with a model where increased expression of $w d r-20$ promotes the catalytic activity of USP-46/WDR-48 complexes resulting in increased surface levels of GLR-1.

\section{USP-46, WDR-48, and WDR-20 localize to endosomes}

We investigated whether USP-46, WDR-48, and WDR-20 localize to internal compartments, such as early and recycling endosomes, where the complex may act to regulate GLR-1 trafficking. We found that mCherry-tagged USP-46, WDR-48, and WDR-20 (under control of the $g l r-1$ promoter) partially overlap with the early endosome marker Venus::Rab-5 in AVA and other GLR-1expressing neuronal soma in the head (Fig. 3A), where they appear to localize at the edges of the Rab-5-labeled compartments. We also found that mCherry-tagged USP-46, WDR-48, and WDR-20 localize to subdomains within compartments labeled by GFP::Syntaxin-13 (Fig. 3B; Chun et al., 2008). Syntaxin13 localizes to early and recycling endosomes and is required for recycling of membrane proteins (Prekeris et al., 1998, 1999). In particular, dominant-negative Syntaxin-13 blocks activity-dependent AMPAR recycling (Park et al., 2004), and AMPARs accumulate in Syntaxin-13-labeled recycling endosomes when the AMPAR recycling protein GRIP-associated protein-1 (GRASP1) is mutated (Chiu et al., 2017). Our data are consistent with USP-46 and the WDR proteins localizing to early and recycling endosomes where they could act to regulate GLR-1 recycling.

\section{USP-46 regulates local insertion of GLR-1}

Given the subcellular localization of USP-46 and the WDR proteins to endosomes, we next examined the mechanism by which USP-46 and the WDR proteins regulate levels of GLR-1 at the cell surface. Ubiquitination of GLR-1 promotes its internalization into endosomes, where the receptors are subsequently sorted either for degradation in the lysosome or recycling back to the plasma membrane (Ehlers, 2000; Burbea et al., 2002). Because deubiquitination of membrane proteins by DUBs at endosomes can rescue them from degradation in the lysosome and promote recycling to the plasma membrane in a variety of cell types (Clague et al., 2012), we tested whether USP-46 acts in the VNC to promote trafficking of receptors from internal compartments to the cell surface. We used dual FRAP of SEP:: mCherry::GLR-1 to measure local insertion of GLR-1 in the VNC, as previously described (Hoerndli et al., 2015). Briefly, 
A SEP::mCherry::GLR-1 in VNC

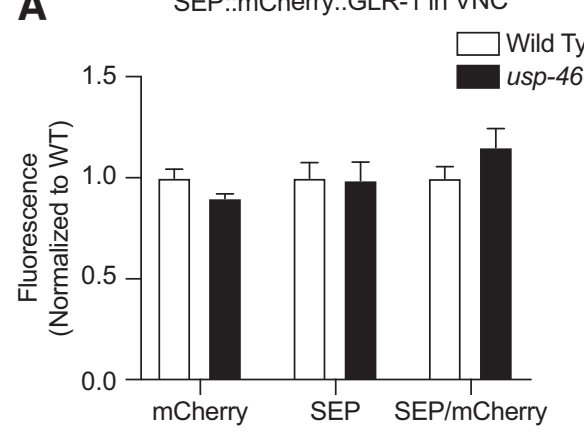

C

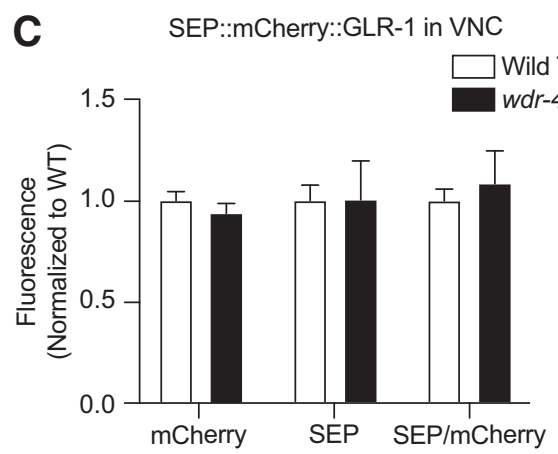

B

SEP::mCherry::GLR-1 in VNC

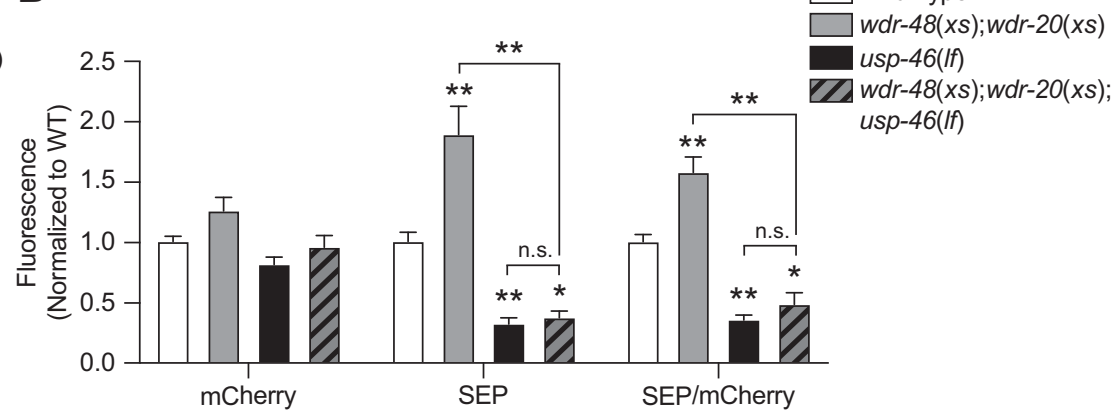

D

SEP::mCherry::GLR-1 in VNC

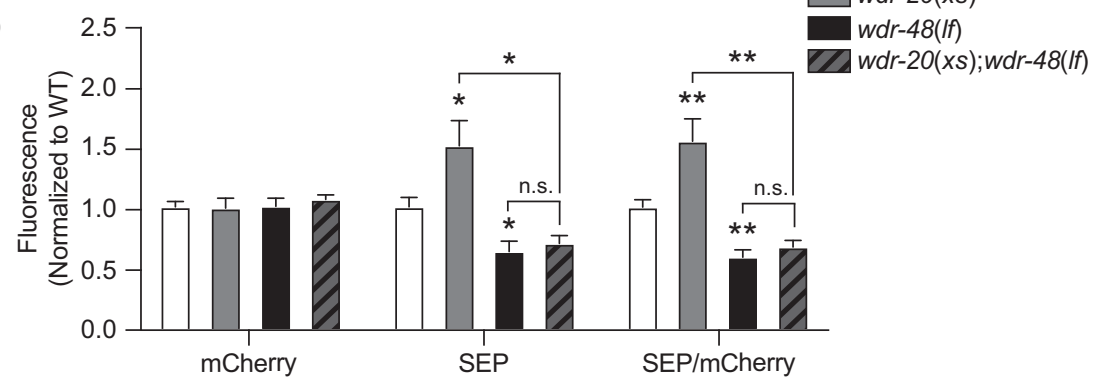

Figure 2. Overexpression of WDR-20 is sufficient to increase GLR-1 surface abundance. A, Quantification of the fluorescence intensity of mCherry (total GLR-1), SEP (surface GLR-1), and the ratio of SEP/mCherry (surface to total GLR-1, normalized to wild type) for animals expressing akls201 in either wild-type $(n=95)$ or animals overexpressing usp-46 cDNA under control of the glr-1 promoter [usp-46(Xs), $n=21]$. Student's $t$ test revealed no significant difference for mCherry $(t=1.043, \mathrm{df}=114, p=0.2992), \operatorname{SEP}(t=0.07,368, \mathrm{df}=114, p=0.9414)$, or SEP $/ \mathrm{mCherry}$ $(t=1.141, \mathrm{df}=114, p=0.2563)$. $\boldsymbol{B}$, Quantification of the fluorescence intensity of mCherry, SEP, and the ratio of SEP/mCherry for animals expressing akls201 in wild type $(n=95)$, animals overexpressing wdr-48 and wdr-20 cDNA under control of the glr-1 promoter [wdr-48(xs);wdr-20(xs), $n=30]$, usp-46 (ok2232) ( $n=69)$ mutants, or animals overexpressing wdr-48 and wdr-20 CDNA under control of the glr-1 promoter in usp-46 (ok2232) mutants [wdr-48(xs);wdr-20(xs);usp-46(Iff), $n=14$ ]. One-way ANOVA revealed a significant effect of genotype for mCherry $\left(F_{(3,204)}=6.006, p=0.0006\right), \operatorname{SEP}\left(F_{(3,204)}=33.67, p<0.0001\right)$, and SEP/mCherry $\left(F_{(3,204)}=45.10, p<0.0001\right)$. C, Quantification of the fluorescence intensity of $\mathrm{mCherry}$, SEP, and the ratio of SEP/mCherry for animals expressing akls201 in wild type $(n=95)$, animals overexpressing wdr-48 CDNA under control of the glr- 1 promoter $(w d r-48(x s), n=30)$. Student's $t$ test revealed no significant difference for mCherry $(t=0.7385, \mathrm{df}=123, p=0.4616)$, SEP $(t=0.02,660, \mathrm{df}=123, p=0.9788)$, or SEP/mCherry $(t=0.6085, \mathrm{df}=123, p=0.5440)$. $D$, Quantification of the fluorescence intensity of mCherry, SEP, and the ratio of SEP/mCherry for animals expressing akls201 in wild type $(n=95)$, animals overexpressing wdr-20 cDNA under control of the glr-1 promoter [wdr-20 (xs), $n=36$; wdr-48 (tm4575), $n=49$ ] mutants, or animals overexpressing wdr-20 CDNA under control of the glr-1 promoter in wdr-48 (tm4575) mutants [wdr-20(xs);wdr-48 $(I f), n=11]$. One-way ANOVA revealed no significant effect of genotype for mCherry $\left(F_{(3,187)}=0.07,553, p=0.9731\right)$, but a significant effect of genotype for $S E P\left(F_{(3,187)}=8.226\right.$, $p<0.0001)$, and SEP/mCherry $\left(F_{(3,187)}=15.05, p<0.0001\right)$. All graphs show mean \pm SEM. Values that differ significantly from the wild type $(\boldsymbol{A}, \boldsymbol{C}$, Student's $t$ test; $\boldsymbol{B}, \boldsymbol{D}$, ANOVA, Tukey's multiple comparison tests) are indicated as follows: $n$.s. $p>0.05, * p \leq 0.01, * * p \leq 0.001$.

mCherry fluorescence is photobleached at the cell surface and in endosomes. Thus, any recovery of mCherry fluorescence after photobleaching can likely be attributed to receptors that have either been newly synthesized or trafficked into the photobleached area. In contrast, because SEP is only photobleached at the cell surface and not in endosomes where it is quenched, recovery of SEP fluorescence after photobleaching represents GLR-1 inserted into the plasma membrane from internal compartments in addition to newly synthesized or trafficked receptors. The difference between the rate of mCherry and SEP fluorescence recovery can be used to estimate the rate of GLR-1 insertion into the plasma membrane from local compartments.

Consistent with a prior study (Hoerndli et al., 2015), we found that $10 \mathrm{~min}$ after photobleaching, SEP fluorescence recovers to $\sim 35-40 \%$ of prebleach levels, whereas mCherry fluorescence recovers to $\sim 10 \%$ of prebleach levels in the VNC of wildtype animals (Fig. 4A,C-E). Interestingly, in usp-46 loss-of-function mutants, SEP fluorescence only recovers to $\sim 10 \%$ of prebleach levels after $10 \mathrm{~min}$ (WT SEP vs $u s p-46$ SEP, $p<0.0001$; Fig. $4 B, C$ ). This low level of recovery is comparable to that of mCherry recovery in $u s p-46$ mutants ( $\sim 7 \%$ after $10 \mathrm{~min})$ suggesting that any remaining recovery of surface GLR-1 abundance in usp-46 mutants is likely due to either non-bleached GLR-1 trafficking into the photobleached area or newly synthesized receptors. Together, these data suggest that USP-46 is required for local GLR-1 insertion into the plasma membrane in the VNC. Similarly, we found that $w d r-48(p<0.0001)$ and $w d r-20$ $(p<0.0001)$ loss-of-function mutants exhibited decreased rates of SEP FRAP but maintained wild-type rates of mCherry fluorescence recovery (Fig. $4 D, E$ ). Conversely, we found that overexpression of $w d r-20$ increases the rate of SEP FRAP $(p<0.0001)$ in a $w d r$-48-dependent manner (Fig. $4 F, G$ ), suggesting that expression of $w d r-20$ is sufficient to promote GLR-1 trafficking to the cell surface from local compartments and its function depends on endogenous $w d r-48$. Finally, overexpression of $w d r-$ 48 and $w d r-20$ also increases the rate of SEP FRAP $(p<0.0001)$ in a usp-46-dependent manner (Fig. $4 H$ ). Collectively, these data indicate that USP-46, WDR-48, and WDR-20 regulate local insertion of GLR-1 into the plasma membrane from internal compartments, consistent with a role for the USP-46/WDR complex in promoting GLR-1 recycling.

$u s p-46, w d r-48$, and $w d r-20$ are required for GLR-1-mediated behavior

Mechanical touch to the nose of the worm induces a locomotion reversal response away from the stimulus that is dependent on the presynaptic vesicular glutamate transporter eat-4/VGLUT (Berger et al., 1998) and glr-1 (Hart et al., 1995; Maricq et al., 
A

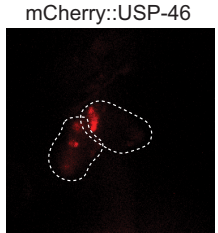

mCherry::WDR-48

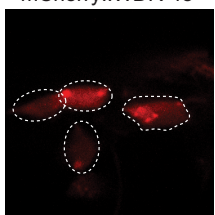

mCherry::WDR-20

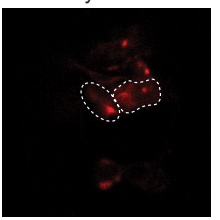

venus::RAB-5

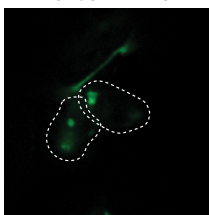

venus::RAB-5

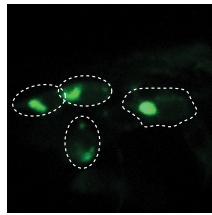

venus::RAB-5

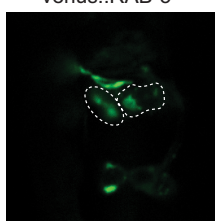

Merge

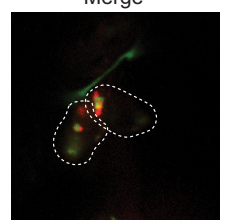

Merge

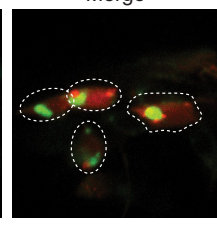

Merge

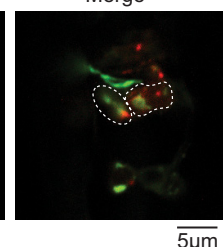

B
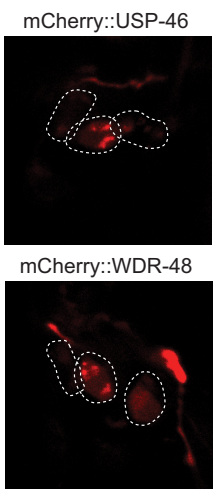

mCherry::WDR-20

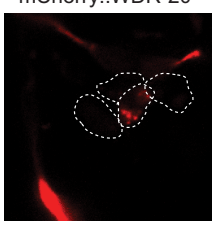

GFP :-Syntaxin-13
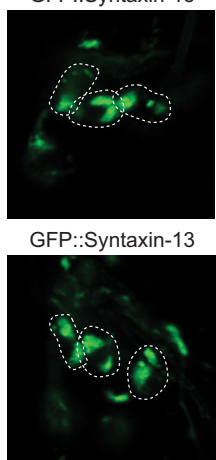

GFP::Syntaxin-13

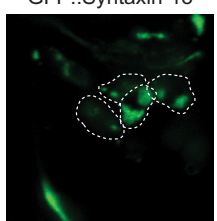

Merge

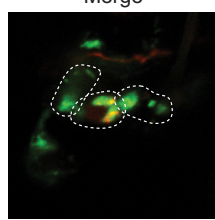

Merge

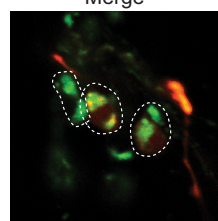

Merge

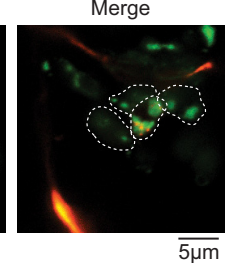

Figure 3. USP-46, WDR-20, and WDR-48 colocalize with endosomal markers. $\boldsymbol{A}$, Representative single plane confocal images of mCherry::USP-46 (pzEx93), mCherry::WDR-48 (pzEx274), or mCherry::WDR-20 (pzEx264) with Venus::Rab-5 (pzEx135) in GLR-1-expressing neuronal cell bodies in the head. $\boldsymbol{B}$, Representative single plane confocal images of mCherry::USP-46 (pzEx93), mCherry::WDR-48 (pzEx274), or mCherry::WDR-20 (pzEx264) with GFP::Syntaxin-13 (nuls232) in GLR-1-expressing neuronal cell bodies in the head. Cell body boundaries are marked by the dotted lines. Fluorescence from co-injection markers is sometimes visible in regions surrounding the cells of interest.

1995). We tested whether the decreased insertion and surface levels of GLR-1 observed in usp-46, $w d r-48$, and $w d r-20$ loss-offunction mutants affected GLR-1 function by analyzing this response. We found that $u s p-46(p<0.0001), w d r-48(p<$ $0.0001)$ and $w d r-20(p<0.0001)$ mutants have comparable defects in the nose-touch response (Fig. 5A). Together with our imaging results, these data suggest that $u s p-46, w d r-48$, and $w d r$ 20 mutants have reduced surface levels of GLR-1 and corresponding defects in glutamatergic nose-touch behavior.

Chronic changes in synaptic activity regulates GLR-1 surface abundance by controlling $w d r-20$ expression

Regulation of AMPARs at the synaptic surface via recycling is a critical mechanism underlying several forms of synaptic plasticity, including homeostatic plasticity (Turrigiano, 2008; Davis, 2013; Huganir and Nicoll, 2013). Previous studies in mammals showed that chronic loss or gain of synaptic activity results in a homeostatic compensatory increase or decrease, respectively, in AMPARs at the synaptic surface (Lissin et al., 1998; O'Brien et al., 1998; Turrigiano et al., 1998; Desai et al., 2002; Turrigiano, 2008). In C. elegans, chronic loss of synaptic activity observed in eat-4/VGLUT loss-of-function mutants results in a homeostaticlike compensatory increase in the total abundance of GLR-1:: GFP in the VNC (Grunwald et al., 2004). This increase in total GLR-1 in the VNC of eat-4 mutants correlates with increased GLR-1 function in the NR because pressure application of glutamate near the NR results in increased glutamate-gated currents in AVA interneurons (Grunwald et al., 2004). We analyzed SEP:: mCherry::GLR-1 levels in the NR to test whether this increase in GLR-1 function was because of an increase in surface levels of GLR-1. We found that eat-4 mutants exhibit increased mCherry fluorescence (total GLR-1, $p<0.0001$ ), consistent with previous findings (Grunwald et al., 2004). Interestingly, we also found that eat-4 mutants exhibit increased SEP fluorescence (surface GLR$1, p<0.0001$ ) in the NR (straightened; Fig. 5E-G), likely underlying the increase in glutamate-evoked currents previously measured. Together, these data indicate that a chronic decrease in synaptic activity results in a homeostatic compensatory increase in surface and total levels of GLR-1.

We next tested whether USP-46 mediates the increase in surface GLR-1 observed in eat-4 mutants by analyzing SEP:: mCherry::GLR-1 in the NR of eat-4;usp-46 double mutants. Consistent with our results in the VNC, we found that $u s p-46$ mutants have decreased SEP fluorescence $(p<0.0001)$ and a decreased SEP/mCherry ratio $(p<0.0001)$ in the NR (Fig. $5 E, F)$. Interestingly, we found that the increase in surface GLR-1 and total GLR-1 observed in eat-4 mutants is suppressed in eat-4; usp-46 double mutants ( $\mathrm{SEP} / \mathrm{mCherry}$ ratio: eat-4 vs eat-4;usp46: $p=0.0041$; usp-46 vs eat-4;usp-46: $p=0.1645$; Fig. 5E,F). These data suggest that the homeostatic compensatory increase in surface GLR-1 abundance observed in eat-4 mutants depends on $u s p-46$.

Because we found that chronic activity-blockade and increased expression of $w d r-20$ are both sufficient to promote surface GLR-1 levels (Figs. 2, 5), we hypothesized that chronic activity-blockade may regulate surface GLR-1 by increasing expression of $w d r-20$. To test this idea, we measured $w d r-20$ expression in eat- 4 mutants using a $w d r-20$ transcriptional reporter (by expressing NLS-GFPLacZ under control of the $w d r-20$ promoter; Dahlberg and Juo, 2014). Because $w d r-20$ is expressed in many neurons (Dahlberg and Juo, 2014), we marked GLR-1-expressing command interneurons with Pnmr-1::dsRed (Brockie et al., 2001), and measured GFP fluorescence ( $P w d r-20$ transcriptional reporter) in the nucleus of the GLR-1-expressing command interneuron AVA (Fig. $5 B)$. Intriguingly, we found that $w d r-20$ transcriptional reporter activity is increased $\sim 62 \%(p=0.0010$ ) in eat-4 mutants (Fig. $5 C$ ). Expression of Pnmr-1::dsRED was not altered in eat-4 mutants $(p=0.8619)$, suggesting that there is not a general upregulation of transcription in these chronic-activity blockade mutants (Fig. 5D). We next tested whether the effects of eat-4 mutation on GLR-1 surface levels were dependent on $w d r-20$. Consistent with our results in the $\mathrm{VNC}$, we found that $w d r-20$ mutants exhibit 
A
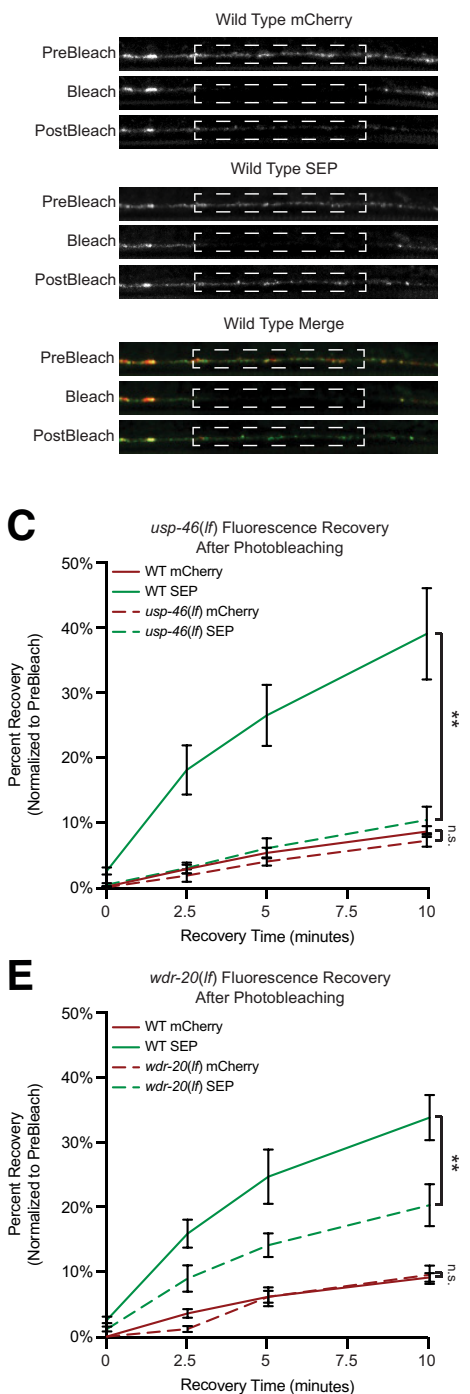

G $w d r-20(x s) ; w d r-48(I f)$ Fluorescence Recovery

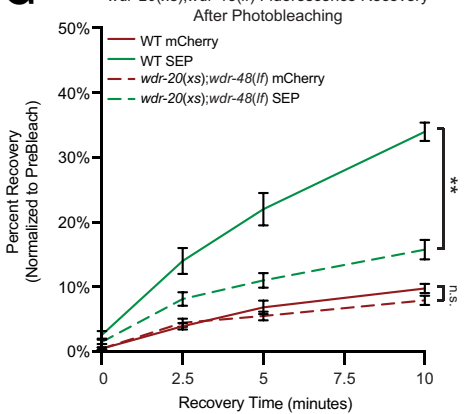

B
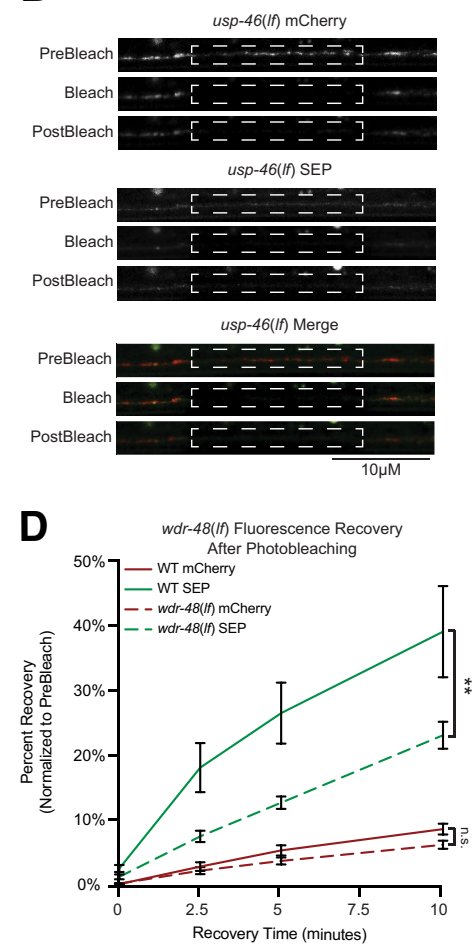

$\mathbf{F}$

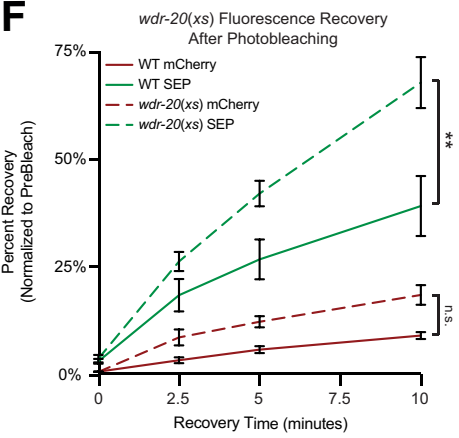

H

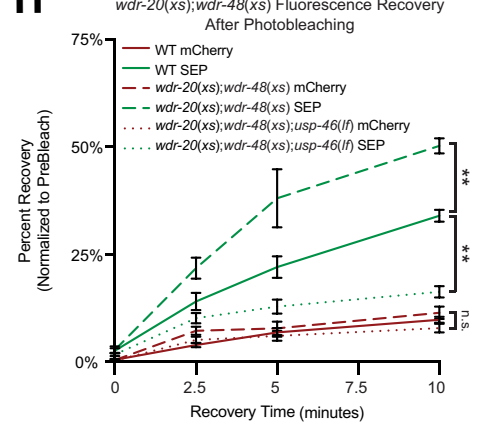

Figure 4. USP-46 is required for local insertion of GLR-1. USP-46 is required for local recycling of GLR-1. $\boldsymbol{A}, \boldsymbol{B}$, Representative prebleach, bleach, and 10-min recovery images of SEP::mCherry::GLR-1 in the VNCS of $L 4$ stage larval animals harboring a SEP::mCherry::GLR-1 integrated transgene (akls201) in either wild-type $(\boldsymbol{A})$ or usp-46 (ok2232) mutants (B). C, Quantification of the rate of recovery for SEP and mCherry (normalized to prebleach intensities) of animals expressing the integrated strain akls201 in either wild-type $(n=10)$ or usp-46 (ok2232) $(n=5)$ mutants. Two-way ANOVA revealed a significant effect of genotype $\left(F_{(3,104)}=14.61\right.$, $p<0.0001)$ and time point $\left(F_{(3,104)}=34.31, p<0.0001\right)$. D, Quantification of the rate of recovery for SEP and $m$ Cherry (normalized to prebleach intensities) of animals expressing the integrated strain ak/s201 in either wildtype $(n=10)$ or or $w d r-48$ (tm4575) $(n=10)$ mutants. The same wild-type data are presented in $\mathbf{C}, \boldsymbol{D}$. Twoway ANOVA revealed a significant effect of genotype $\left(F_{(3,104)}=53.75, p<0.0001\right)$ and time point $\left(F_{(3,160)}=\right.$ $45.59, p<0.0001)$. $\boldsymbol{E}$, Quantification of the rate of recovery for SEP and mCherry (normalized to prebleach intensities) of animals expressing the integrated strain pzls45 in either wild-type $(n=5)$ or wdr-20 ( $g k 547140)$ $(n=5)$ mutants. Two-way ANOVA revealed a significant effect of genotype $\left(F_{(3,64)}=53.29, p<0.0001\right)$ and time point $\left(F_{(3,64)}=58.97, p<0.0001\right)$. $\boldsymbol{F}$, Quantification of the rate of recovery for SEP and mCherry

decreased SEP fluorescence $(p=0.0138)$ in the NR (Fig. 5F,G). Interestingly, we found that the increased mCherry and SEP fluorescence observed in eat-4 mutants was also prevented in $w d r-20$ mutants (SEP/ mCherry ratio: eat-4 vs eat-4;wdr-20: $p=0.0054$; $w d r-20$ vs eat-4; $w d r-20: p=0.9484$; Fig. $5 F, G$ ), suggesting that increases in surface and total levels of GLR-1 induced by chronic activity-blockade require $w d r-20$. Together, these data support a model where chronic loss of activity (i.e., in eat-4/VGLUT mutants) increases expression of $w d r$-20, resulting in activation of USP-46 and increased recycling of GLR-1 to the cell surface.

We hypothesized that a chronic increase in glutamate signaling might have the opposite effect of eat-4 mutants and lead to decreased surface levels of GLR-1. In C. elegans, there are six glutamate transporters (GluTs) that are thought to be involved in clearing glutamate from the extracellular space. Mutants lacking one or more of these glt/GluT genes exhibit alterations in glutamatergic behaviors that are consistent with increased extracellular glutamate and enhanced glutamate signaling (Mano et al., 2007). Interestingly, we found that mutants lacking glt-3, glt-6, and glt-7 exhibited reduced surface GLR-1 levels ( $p=0.0061$; Fig. $5 H$ ), consistent with the notion that chronic increases in glutamate signaling results in a compensatory reduction in surface levels of GLR-1. We also found that $w d r-20$ expression was reduced in glt-3 glt-6 glt-7 loss-offunction mutants $(p=0.0459)$ based on a $w d r-20$ transcriptional reporter (Fig. $5 B, C$ ). Since we showed that loss of $w d r-20$ results in reduced surface levels of GLR-1, we tested whether overexpression of $w d r-20$ could counteract the effects of glt-3 glt-6 glt-7 loss-offunction on surface levels of GLR-1. Intriguingly, we found that overexpression of $w d r$-20 blocks the reduced GLR-1 surface levels observed in glt-3 glt-6 glt-7 mutants [SEP/mCherry ratio: glt-3 glt-6 glt-7 vs

$\leftarrow$

(normalized to prebleach intensities) of animals expressing the integrated strain akls201 in either a wild-type background $(n=10)$ or animals overexpressing $w d r-20$ under control of the glr-1 promoter [ $w d r-20$ $(x s), n=6]$. Two-way ANOVA revealed a significant effect of genotype $\left(F_{(3,112)}=61.69, p<0.0001\right)$ and time point $\left(F_{(3,112)}=61.69\right.$, $p<0.0001)$. G, Quantification of the rate of recovery for SEP and $\mathrm{mCherry} \mathrm{(normalized} \mathrm{to} \mathrm{prebleach} \mathrm{intensities)} \mathrm{of} \mathrm{animals} \mathrm{expressing}$ the integrated strain ak/s201 in either a wild-type background $(n=13)$ or animals overexpressing $w d r-20$ under control of the glr-1 promoter in a $w d r-48(t m 4575)$ mutant background [ $w d r-20(x s) ; w d r-48(I f), n=3]$. Two-way ANOVA revealed a significant effect of genotype $\left(F_{(3,192)}=\right.$ 119.0, $p<0.0001)$ and time point $\left(F_{(3,192)}=130.9, p<0.0001\right)$. $\boldsymbol{H}$, Quantification of the rate of recovery for SEP and mCherry (normalized to prebleach intensities) of animals expressing the integrated strain akls201 in either a wild-type background $(n=13)$, animals overexpressing $w d r-20$ and $w d r-48$ under control of the glr- 1 promoter $[w d r-20(x s)$; $w d r-48(x s), n=5$ ] or animals overexpressing $w d r-20$ and $w d r-48$ under control of the glr-1 promoter in a usp-46 (ok2232) mutant background [wdr-20(xs);wdr-48(xs);usp-46(If), $n=11]$. Two-way ANOVA revealed a significant effect of genotype $\left(F_{(3,208)}=114.3, p<0.0001\right)$ and time point $\left(F_{(3,208)}=165.4, p<0.0001\right)$. All graphs show mean \pm SEM. Values that differ significantly from the wild-type (ANOVA, Tukey's multiple comparison tests) are indicated as follows: n.s. $p>0.05, * * p \leq 0.001$. 
A

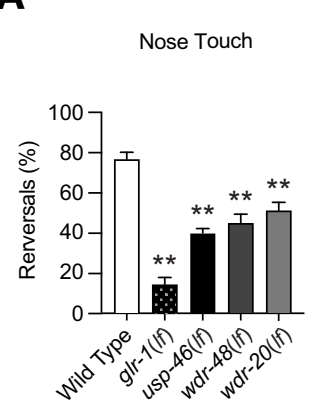

B

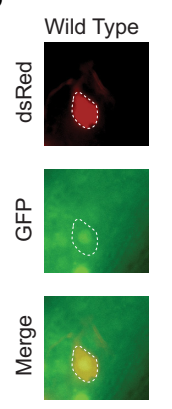

$g \mid t-3(I f) g l t-6(I f)$

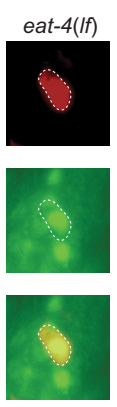

E

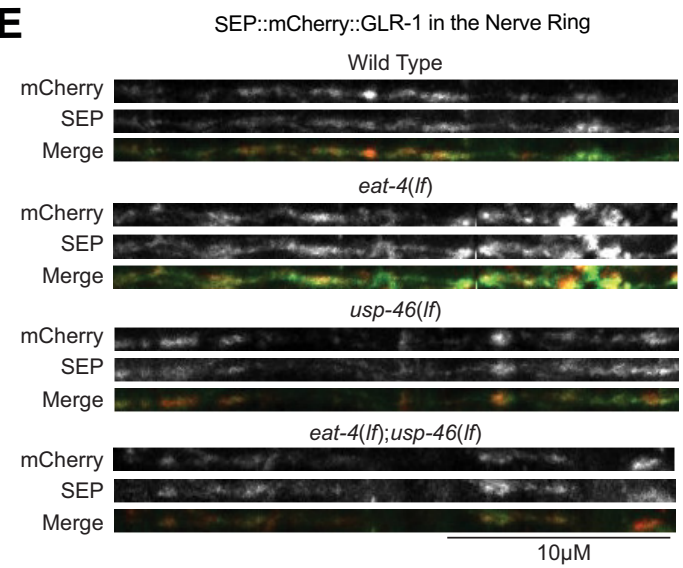

\section{G}

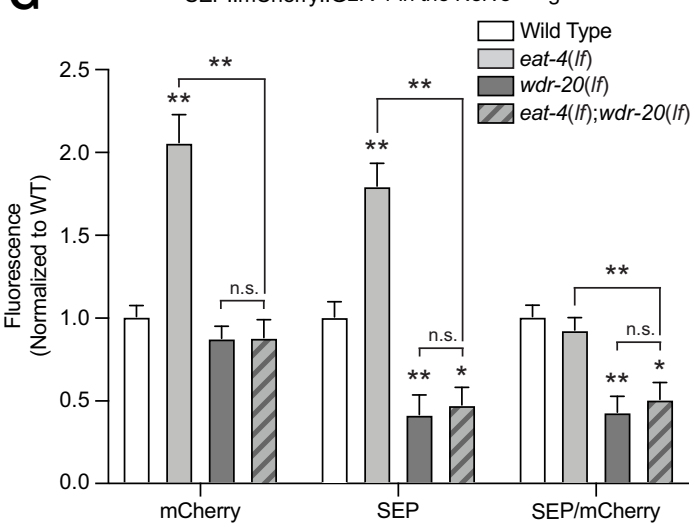

C
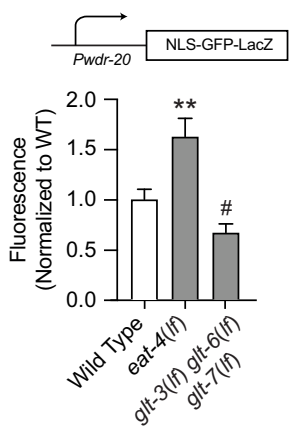

D

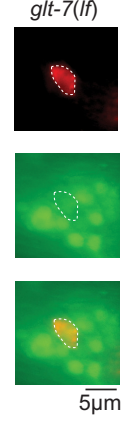

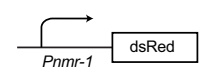

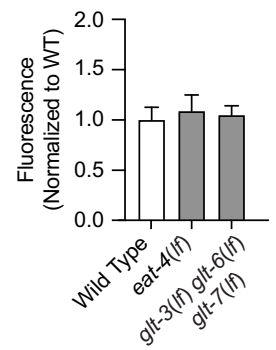

F
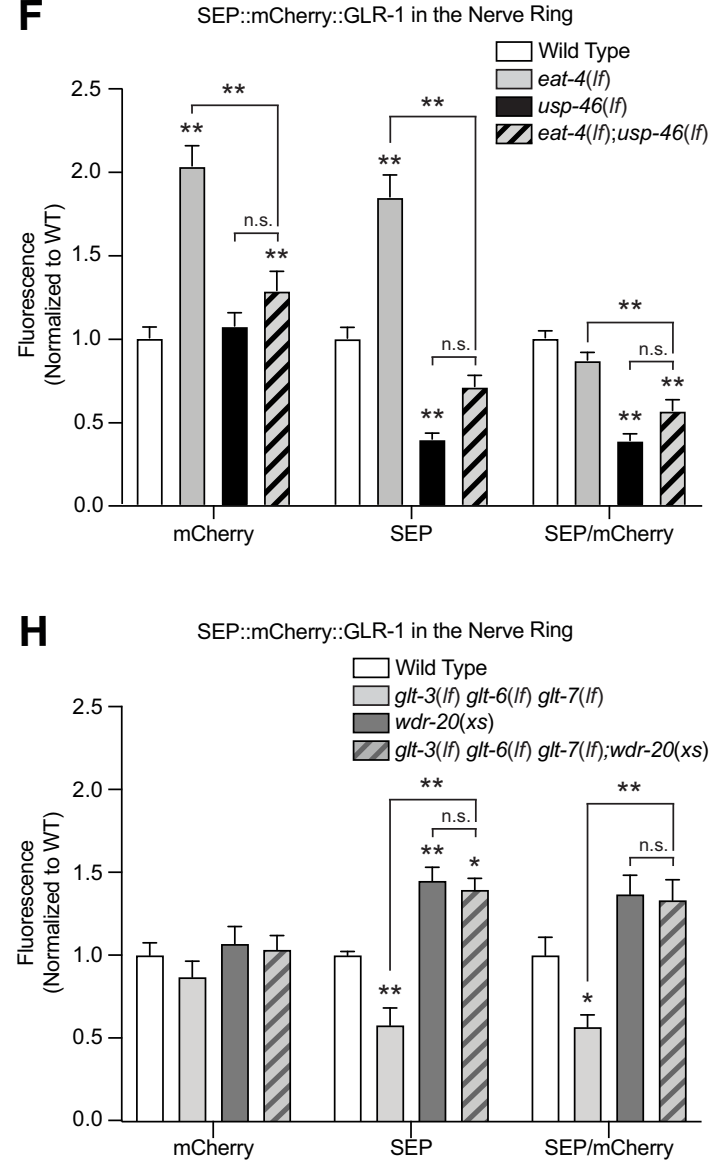

Figure 5. Chronic activity-blockade promotes GLR-1 surface levels in the same pathway as WDR-20 and USP-46. A, Quantification of the nose-touch response for wild-type ( $n=16)$, and glr1 (n2461) $(n=16)$, usp-46 (ok2232) $(n=15)$, wdr-48 (tm4575) $(n=16)$, or wdr-20 $(g k 547140)(n=16)$ mutants. One-way ANOVA revealed a significant effect of genotype $\left(F_{(4,74)}=39.46\right.$, $p<0.0001$ ). B. Representative images of NLS-GFP-LacZ in the nucleus of AVA (marked by Pnmr-1::dsRED) of L4 stage larval animals harboring an integrated transgene (pz/s38) that expresses NLS-GFP-LacZ under control of the wdr-20 promoter (Pwdr-20) in either wild-type $(n=11)$, eat-4(n2474) $(n=15)$, or glt-3 (bz34) glt-6 $(t m 1316)$ glt-7 (tm1641) $(n=14)$ mutants. C, Quantification of GFP intensity (Pwdr-20::NLS-GFP-LacZ) in the AVA nucleus for the strains described in $\boldsymbol{B}$. One-way ANOVA revealed a significant effect of genotype $\left(F_{(2,50)}=15.00\right.$, $p<0.0001)$. D, Quantification of dsRED intensity $(P n m r-1:: d s R E D)$ in AVA for the strains described in $\boldsymbol{B}$. One-way ANOVA revealed no significant effect of genotype $\left(F_{(2,50)}=0.1135\right.$, $p=0.8929)$. $\boldsymbol{E}$, Representative images of SEP:::mCherry::GLR-1 in the NRs (straightened) of L4 stage larval animals harboring a SEP:::mCherry::GLR-1 integrated transgene (akls201) in either wild-type $(n=52)$ animals, eat-4 (n2474) ( $n=22)$, usp-46 (ok2232) ( $n=25)$, or eat-4 (n2474);usp-46 (ok2232) $(n=15)$ mutants. $\boldsymbol{F}$, Quantification of the fluorescence intensity of mCherry (total GLR-1), SEP (surface GLR-1), and the ratio of SEP/mCherry (surface to total GLR-1, normalized to wild type) for strains described in $\boldsymbol{E}$. One-way ANOVA revealed a significant effect of genotype for mCherry $\left(F_{(3,109)}=25.92, p<0.0001\right)$, SEP $\left(F_{(3,109)}=47.22, p<0.0001\right)$, and SEP/mCherry $\left(F_{(3,109)}=35.36, p<0.0001\right)$. G, Quantification of the fluorescence intensity of $m$ Cherry (total GLR-1), SEP (surface GLR-1), and the ratio of SEP/mCherry (surface to total GLR-1, normalized to wild type) of SEP::mCherry::GLR-1 in the NRs (straightened) of L4 stage larval animals harboring a SEP::mCherry:GLR-1 integrated transgene (pzls45) in either wild-type $(n=20)$ animals, eat-4 (n2474) $(n=21)$, wdr-20 $(g k 547140)(n=10)$, or eat-4 $(n 2474) ; w d r-20$ ( $g$ k547140) $(n=11)$ mutants. One-way ANOVA revealed a significant effect of genotype for mCherry $\left(F_{(3,58)}=21.93, p<0.0001\right)$, SEP $\left(F_{(3,58)}=27.22, p<0.0001\right)$, and SEP/mCherry $\left(F_{(3,58)}=11.07, p<0.0001\right)$. $\boldsymbol{H}$, Quantification of the fluorescence intensity of mCherry (total GLR-1), SEP (surface GLR-1), and the ratio of SEP/mCherry (surface to total GLR-1, normalized to wild type) of SEP:::mCherry::GLR-1 in the NRs (straightened) of L4 stage larval animals harboring a SEP::mCherry::GLR-1 integrated transgene (akls201) in either wild-type $(n=12)$ animals, glt3 (bz34) glt-6 (tm1316) glt-7 (tm1641) ( $n=19)$, animals overexpressing wdr-20 CDNA under control of the glr-1 promoter [wdr-20 (xs), $n=15]$, or animals overexpressing wdr-20 cDNA under control of the glr-1 promoter in glt-3 (bz34) glt-6 (tm1316) glt-7 (tm1641) mutants (wdr-20(xs); glt-3 (bz34) glt-6 (tm1316) glt-7 (tm1641) ( $n=13)$. One-way ANOVA revealed no significant effect of genotype for mCherry $\left(F_{(3,55)}=1.011, p=0.3949\right)$, but a significant effect of genotype for SEP $\left(F_{(3,55)}=24.63, p<0.0001\right)$ and SEP/mCherry $\left(F_{(3,55)}=15.08, p<0.0001\right)$. All graphs shown mean \pm SEM. Values that differ significantly from the wild type (ANOVA, Tukey's multiple comparisons tests) are indicated as follows: $n . S . p>0.05, \# p \leq 0.05, * p \leq 0.01$, $* * p \leq 0.001$. 
$w d r-20(x s) ;$ glt-3 glt-6 glt-7: $p<0.0001 ; w d r-20(x s)$ vs $w d r-20(x s)$; glt-3 glt-6 glt-7: $p=0.9948$; Fig. $5 \mathrm{H}$ ]. Collectively, these data suggest a model where chronic reduction or increase in glutamate signaling bidirectionally regulates $w d r-20$ expression and surface levels of GLR-1.

\section{Discussion}

Activity-dependent regulation of AMPAR recycling from endosomes to the synaptic surface contributes to the control of synaptic strength (Huganir and Nicoll, 2013). The reversible modification of membrane proteins by ubiquitin is one mechanism known to regulate membrane protein recycling in multiple cell types (Clague et al., 2012). Although the covalent attachment of ubiquitin to AMPARs decreases their surface levels and promotes receptor degradation (Goo et al., 2015), the role of ubiquitin removal in controlling AMPAR recycling has not been investigated. We previously showed that deubiquitination of GLR-1 by the USP-46/WDR complex prevents GLR-1 degradation in the lysosome (Dahlberg and Juo, 2014). We also found that overexpression of WDR-20 and WDR-48 increases surface levels of GLR-1 in cultured C. elegans embryonic neurons. Here, we used GLR-1-tagged with $\mathrm{pH}$-sensitive SEP and mCherry (SEP::mCherry::GLR-1) to show that USP-46, WDR-48, and WDR-20, act cell-autonomously to promote GLR-1 surface levels in vivo (Fig. 1). Loss of usp-46, $w d r-48$, or $w d r-20$ results in decreased surface levels of GLR-1 (Fig. 1) and corresponding defects in GLR-1-mediated behavior (Fig. 5A), suggesting that USP46 regulation of GLR-1 is important for glutamatergic signaling.

Although binding of WDR-48 to USP-46 (or USP-12) stimulates the low intrinsic catalytic activity of the DUB, subsequent interaction with WDR-20 synergistically enhances DUB complex activity (Cohn et al., 2009; Kee et al., 2010; Faesen et al., 2011), suggesting that regulating WDR-20 could provide a mechanism to control USP-46 function. We found that overexpression of usp-46 or $w d r-48$ alone does not increase surface GLR-1 levels in vivo. However, overexpression of $w d r-20$ was sufficient to increase surface GLR-1 levels in vivo (Figs. 2, 5) and to promote local GLR-1 insertion (Fig. 4) in a $u s p-46$ and $w d r-48$-dependent manner. Together, these data are consistent with the idea that increased expression of WDR-20 works together with WDR-48 and USP-46 to promote surface levels of GLR-1 by increasing the rate of local receptor insertion. Because WDR-48 and WDR-20 increase the catalytic activity of USP-46 resulting in the deubiquitination of GLR-1 (Kowalski et al., 2011; Dahlberg and Juo, 2014), these data are consistent with a model whereby WDR-20 promotes GLR-1 surface levels by activating USP-46 to deubiquitinate the receptor.

The regulation of AMPAR levels by ubiquitin and USP46 is conserved in mammals (Goo et al., 2015; Hodul et al., 2017). Consistent with our findings in C. elegans (Kowalski et al., 2011), RNAi knock down of mammalian Usp46 in cultured rodent neurons results in decreased levels of surface and total AMPARs (Huo et al., 2015). Although there is some conflicting data from mammalian neurons regarding whether AMPARs are ubiquitinated at the plasma membrane (Schwarz et al., 2010; Lin et al., 2011) or at endosomes (Lussier et al., 2011; Widagdo et al., 2015), it is clear that ubiquitination of AMPARs can lead to receptor degradation. Several studies suggest that AMPAR ubiquitination results in degradation in the lysosome (Burbea et al., 2002; Chun et al., 2008; Schwarz et al., 2010; Kowalski et al., 2011; Lussier et al., 2011; Widagdo et al., 2015). Consistent with this, mammalian AMPARs are modified primarily by Lys63- linked ubiquitin chains (Huo et al., 2015; Widagdo et al., 2015), which target membrane proteins for degradation in the lysosome (Katzmann et al., 2002; Lauwers et al., 2009), and USP46 has a preference for Lys63-linked ubiquitin chains (Huo et al., 2015).

DUBs control membrane trafficking at multiple organelles. In particular, DUBs act at sorting endosomes to deubiquitinate membrane receptors and divert them toward the recycling pathway and away from the lysosome (Clague et al., 2012). We propose that USP-46 deubiquitinates GLR-1 in endosomes to prevent degradation and to promote receptor recycling. Consistent with this model, a mutant version of GluA1 that has reduced ubiquitination [GluA1(K868R)] is protected from lysosomal degradation and recycles faster to the neuronal cell surface after agonist treatment (Widagdo et al., 2015). While it is thought that ubiquitination targets membrane proteins for lysosomal degradation at the expense of recycling, it is not clear whether deubiquitination of membrane receptors occurs independently at two separate steps to prevent degradation and to promote recycling, especially in cases where the same DUB and substrate ubiquitination sites are involved. A direct role for deubiquitination in insertion and recycling to the plasma membrane has been shown in cases where ubiquitin or the DUB have non-degradative functions. For example, the glucose transporter GLUT4 is targeted to a specialized compartment by ubiquitin and translocates to the plasma membrane in response to insulin (Watson et al., 2004). Similarly, the guidance receptor Robo1, traffics to the cell surface from an internal compartment in a Usp33-dependent manner after activation by its ligand Slit (Yuasa-Kawada et al., 2009a,b). Because Usp33 does not regulate Robol degradation, this study provides a clear example of a DUB specifically regulating receptor insertion separately from degradation. While we cannot distinguish whether the USP-46/ WDR complex acts at one or two locations to prevents GLR-1 degradation and promote recycling, the colocalization of USP46, WDR-48, and WDR-20 within Syntaxin-13-labeled recycling endosome compartments and at the edge of Rab-5-labeled early endosome compartments (Fig. 3), and the stronger effect of usp-46 mutants on surface versus total GLR-1 levels (Fig. 1), could be consistent with the USP-46/WDR complex acting at two separate steps.

Chronic changes in synaptic activity leads to a homeostatic alteration in synaptic strength termed synaptic scaling that can largely be attributed to changes in AMPAR levels at the synaptic membrane (Turrigiano, 2008). In rodent central neurons, synaptic AMPAR levels are upregulated or downregulated in response to chronic activity-blockade (i.e., after TTX treatment) or increased neuronal activity (i.e., after bicuculline treatment), respectively (Turrigiano, 2008). In C. elegans, loss-of-function mutation of eat4/VGLUT leads to a chronic loss of glutamate release resulting in a homeostatic compensatory increase in GLR-1 levels in the VNC and an increase in current amplitudes in response to glutamate application near the NR (Grunwald et al., 2004). We found that this compensatory increase in GLR-1 observed in the VNC of eat-4 mutants also occurs in the NR and results in increased surface and total levels of GLR-1 in a $u s p-46$ and $w d r$-20-dependent manner (Fig. 5E-G). Furthermore, $w d r-20$ transcription was increased in eat-4 mutants (Fig. 5B,C), revealing an activity-dependent mechanism to control $w d r-20$ expression. Conversely, we found that $g l t-3$ glt-6 glt-7/GluT mutants, which are thought to have increased extracellular glutamate (Mano et al., 2007), exhibit decreased $w d r$ 20 expression and surface levels of GLR-1 (Fig. 5H). Intriguingly, overexpression of $w d r-20$ was sufficient to block the reduction in surface GLR-1 in GluT mutants (Fig. 5H). Together, these data are 
consistent with a model whereby chronic decreases or increases in synaptic activity bidirectionally regulates $w d r-20$ expression, which in turn modulates USP-46-dependent deubiquitination of GLR-1 to control the rate of local insertion and surface levels.

While the mechanism by which chronic changes in synaptic activity regulates $w d r-20$ expression is unknown, we hypothesize that alterations in calcium influx mediated by GLR-1, which is calcium-permeable (Zheng et al., 1999), or voltage-gated calcium channels, regulate calcium-responsive signaling pathways and downstream transcription factors to control $w d r-20$ transcription. In this scenario, reduced intracellular calcium in eat-4/ VGLUT mutants would lead to decreased activation of calciumresponsive transcription factors such as $\mathrm{CRH}-1 / \mathrm{cAMP}$ response element-binding protein (CREB) resulting in increased transcription of $w d r-20$. Given that mammalian and C. elegans $w d r-$ 20 promoters contain consensus CREB binding sites (Zhang et al., 2005), CRH-1/CREB may directly repress $w d r-20$ transcription in a non-canonical manner. Alternatively, CRH-1/CREB may indirectly repress $w d r-20$ transcription by activating a repressor. It will be informative to test whether CRH-1/CREB and upstream signaling components, such as CaM kinases, mediate the effect of chronic activity mutants on $w d r-20$ expression.

In summary, we identified a novel, activity-dependent mechanism in C. elegans where increased expression of $w d r$-20 activates USP-46 to control AMPAR trafficking and surface levels. We speculate that $w d r$-20 expression may also be regulated during mammalian homeostatic plasticity as a mechanism to control AMPAR surface levels via USP-46. Future studies will be required to test whether the chronic activity-dependent changes we observe in our genetic mutant models also occur in mammalian neurons after manipulations that induce homeostatic upscaling or downscaling of AMPARs. Interestingly, the DUB USP8 has been implicated in regulating homeostatic downscaling of AMPARs in cultured mammalian neurons (Scudder et al., 2014). Thus, different upstream signals may couple to specific DUBs or ubiquitin ligases to control the ubiquitin status and trafficking fate of AMPARs.

Emerging evidence suggests that dysregulation of AMPAR ubiquitination and endosomal recycling pathways may contribute to neurodegenerative and neurologic disorders ( $\mathrm{Li}$ and DiFiglia, 2012; Widagdo et al., 2017). Soluble oligomeric forms of amyloid- $\beta$ can induce synaptic depression, which is correlated with increased ubiquitination and downregulation of AMPARs (Rodrigues et al., 2016; Guntupalli et al., 2017). Additionally, point mutations in the endosomal protein GRIPassociated protein-1 (GRASP1) found in patients with intellectual disability, disrupt its function in AMPAR recycling and hippocampal plasticity (Chiu et al., 2017), providing a direct link between defects in AMPAR recycling and intellectual disability. Finally, since USP-46 and the WDR proteins are highly conserved from yeast to mammals (Hodul et al., 2017) and over a third of all USP family DUBs interact with WDR proteins (Sowa et al., 2009), our finding that synaptic activity can regulate WDR-20 expression to control USP-46 may be broadly informative for elucidating how other WDR proteins are regulated to control their cognate DUBs in both neuronal and non-neuronal cells.

\section{References}

Anggono V, Huganir RL (2012) Regulation of AMPA receptor trafficking and synaptic plasticity. Curr Opin Neurobiol 22:461-469.

Berger AJ, Hart AC, Kaplan JM (1998) G alphas-induced neurodegeneration in Caenorhabditis elegans. J Neurosci 18:2871-2880.
Bingol B, Sheng M (2011) Deconstruction for reconstruction: the role of proteolysis in neural plasticity and disease. Neuron 69:22-32.

Brenner S (1974) The genetics of Caenorhabditis elegans. Genetics 77:71-94.

Brockie PJ, Madsen DM, Zheng Y, Mellem J, Maricq AV (2001) Differential expression of glutamate receptor subunits in the nervous system of Caenorhabditis elegans and their regulation by the homeodomain protein UNC-42. J Neurosci 21:1510-1522.

Burbea M, Dreier L, Dittman JS, Grunwald ME, Kaplan JM (2002) Ubiquitin and AP180 regulate the abundance of GLR-1 glutamate receptors at postsynaptic elements in C. elegans. Neuron 35:107-120.

Chiu SL, Diering GH, Ye B, Takamiya K, Chen CM, Jiang Y, Niranjan T, Schwartz CE, Wang T, Huganir RL (2017) GRASP1 regulates synaptic plasticity and learning through endosomal recycling of AMPA receptors. Neuron 93:1405-1419.e8.

Chun DK, McEwen JM, Burbea M, Kaplan JM (2008) UNC-108/Rab2 regulates postendocytic trafficking in Caenorhabditis elegans. Mol Biol Cell 19:2682-2695.

Clague MJ, Coulson JM, Urbé S (2012) Cellular functions of the DUBs. J Cell Sci 125:277-286.

Clague MJ, Urbé S, Komander D (2019) Breaking the chains: deubiquitylating enzyme specificity begets function. Nat Rev Mol Cell Biol 20:338352.

Cohn MA, Kee Y, Haas W, Gygi SP, D'Andrea AD (2009) UAF1 is a subunit of multiple deubiquitinating enzyme complexes. J Biol Chem 284:53435351.

Dahlberg CL, Juo P (2014) The WD40-repeat proteins WDR-20 and WDR48 bind and activate the deubiquitinating enzyme USP-46 to promote the abundance of the glutamate receptor GLR-1 in the ventral nerve cord of Caenorhabditis elegans. J Biol Chem 289:3444-3456.

Davis GW (2013) Homeostatic signaling and the stabilization of neural function. Neuron 80:718-728.

Desai NS, Cudmore RH, Nelson SB, Turrigiano GG (2002) Critical periods for experience-dependent synaptic scaling in visual cortex. Nat Neurosci 5:783-789.

Dreier L, Burbea M, Kaplan JM (2005) LIN-23-mediated degradation of $\beta$-catenin regulates the abundance of GLR- 1 glutamate receptors in the ventral nerve cord of C. elegans. Neuron 46:51-64.

Ehlers MD (2000) Reinsertion or degradation of AMPA receptors determined by activity-dependent endocytic sorting. Neuron 28:511-525.

Faesen AC, Luna-Vargas MP, Geurink PP, Clerici M, Merkx R, van Dijk WJ, Hameed DS, El Oualid F, Ovaa H, Sixma TK (2011) The differential modulation of USP activity by internal regulatory domains, interactors and eight ubiquitin chain types. Chem Biol 18:1550-1561.

Fu AK, Hung KW, Fu WY, Shen C, Chen Y, Xia J, Lai KO, Ip NY (2011) APC(Cdh1) mediates EphA4-dependent downregulation of AMPA receptors in homeostatic plasticity. Nat Neurosci 14:181-189.

Goo MS, Scudder SL, Patrick GN (2015) Ubiquitin-dependent trafficking and turnover of ionotropic glutamate receptors. Front Mol Neurosci 8:60

Grunwald ME, Mellem JE, Strutz N, Maricq AV, Kaplan JM (2004) Clathrinmediated endocytosis is required for compensatory regulation of GLR-1 glutamate receptors after activity blockade. Proc Natl Acad Sci USA 101:3190-3195.

Guntupalli S, Jang SE, Zhu T, Huganir RL, Widagdo J, Anggono V (2017) GluA1 subunit ubiquitination mediates amyloid- $\beta$-induced loss of surface $\alpha$-amino-3-hydroxy-5-methyl-4-isoxazolepropionic acid (AMPA) receptors. J Biol Chem 292:8186-8194.

Hart AC, Sims S, Kaplan JM (1995) Synaptic code for sensory modalities revealed by C. elegans GLR-1 glutamate receptor. Nature 378:82-85.

Hodul M, Dahlberg CL, Juo P (2017) Function of the deubiquitinating enzyme USP46 in the nervous system and its regulation by WD40-repeat proteins. Front Synaptic Neurosci 9:16.

Hoerndli FJ, Maxfield DA, Brockie PJ, Mellem JE, Jensen E, Wang R, Madsen DM, Maricq AV (2013) Kinesin-1 regulates synaptic strength by mediating the delivery, removal, and redistribution of AMPA receptors. Neuron 80:1421-1437.

Hoerndli FJ, Wang R, Mellem JE, Kallarackal A, Brockie PJ, Thacker C, Madsen DM, Maricq AV (2015) Neuronal activity and CaMKII regulate kinesin-mediated transport of synaptic AMPARs. Neuron 86:457-474.

Hou Q, Gilbert J, Man HY (2011) Homeostatic regulation of AMPA receptor trafficking and degradation by light-controlled single-synaptic activation. Neuron 72:806-818. 
Huganir RL, Nicoll RA (2013) AMPARs and synaptic plasticity: the last 25 years. Neuron 80:704-717.

Huo Y, Khatri N, Hou Q, Gilbert J, Wang G, Man HY (2015) The deubiquitinating enzyme USP46 regulates AMPA receptor ubiquitination and trafficking. J Neurochem 134:1067-1080.

Juo P, Kaplan JM (2004) The anaphase-promoting complex regulates the abundance of GLR-1 glutamate receptors in the ventral nerve cord of $C$. elegans. Curr Biol 14:2057-2062.

Kaplan JM, Horvitz J (1993) A dual mechanosensory and chemosensory neuron in Caenorhabditis elegans. Proc Natl Acad Sci USA 90:2227-2231.

Katzmann DJ, Odorizzi G, Emr SD (2002) Receptor downregulation and multivesicular-body sorting. Nat Rev Mol Cell Biol 3:893-905.

Kee Y, Yang K, Cohn MA, Haas W, Gygi SP, D’Andrea AD (2010) WDR20 regulates activity of the USP 12 x UAF1 deubiquitinating enzyme complex. J Biol Chem 285:11252-11257.

Kouranti I, McLean JR, Feoktistova A, Liang P, Johnson AE, RobertsGalbraith RH, Gould KL (2010) A global census of fission yeast deubiquitinating enzyme localization and interaction networks reveals distinct compartmentalization profiles and overlapping functions in endocytosis and polarity. PLoS Biol 8:e1000471.

Kowalski JR, Dahlberg CL, Juo P (2011) The deubiquitinating enzyme USP46 negatively regulates the degradation of glutamate receptors to control their abundance in the ventral nerve cord of Caenorhabditis elegans. J Neurosci 31:1341-1354.

Lauwers E, Jacob C, André B (2009) K63-linked ubiquitin chains as a specific signal for protein sorting into the multivesicular body pathway. J Cell Biol 185:493-502.

Lauwers E, Erpapazoglou Z, Haguenauer-Tsapis R, André B (2010) The ubiquitin code of yeast permease trafficking. Trends Cell Biol 20:196-204.

Lee SH, Simonetta A, Sheng M (2004) Subunit rules governing the sorting of internalized AMPA receptors in hippocampal neurons. Neuron 43:221236.

Li X, DiFiglia M (2012) The recycling endosome and its role in neurological disorders. Prog Neurobiol 97:127-141.

Lin A, Man HY (2014) Endocytic adaptor epidermal growth factor receptor substrate 15 (Eps15) is involved in the trafficking of ubiquitinated $\alpha$-amino-3-hydroxy-5-methyl-4-isoxazolepropionic acid receptors. J Biol Chem 289:24652-24664.

Lin A, Hou Q, Jarzylo L, Amato S, Gilbert J, Shang F, Man HY (2011) Nedd4-mediated AMPA receptor ubiquitination regulates receptor turnover and trafficking. J Neurochem 119:27-39.

Lissin DV, Gomperts SN, Carroll RC, Christine CW, Kalman D, Kitamura M, Hardy S, Nicoll RA, Malenka RC, von Zastrow M (1998) Activity differentially regulates the surface expression of synaptic AMPA and NMDA glutamate receptors. Proc Natl Acad Sci USA 95:7097-7102.

Lussier MP, Nasu-Nishimura Y, Roche KW (2011) Activity-dependent ubiquitination of the AMPA receptor subunit GluA2. J Neurosci 31:30773081.

Lussier MP, Herring BE, Nasu-Nishimura Y, Neutzner A, Karbowski M, Youle RJ, Nicoll RA, Roche KW (2012) Ubiquitin ligase RNF167 regulates AMPA receptor-mediated synaptic transmission. Proc Natl Acad Sci USA 109:19426-19431.

Luth ES, Hodul M, Rennich BJ, Riccio C, Hofer J, Markoja K, Juo P (2021) VER/VEGF receptors regulate AMPA receptor surface levels and glutamatergic behavior. PLoS Genet 17:e1009375.

Mano I, Straud S, Driscoll M (2007) Caenorhabditis elegans glutamate transporters influence synaptic function and behavior at sites distant from the synapse. J Biol Chem 282:34412-34419.

Maricq AV, Peckol E, Driscoll M, Bargmann CI (1995) Mechanosensory signalling in C. elegans mediated by the GLR-1 glutamate receptor. Nature 378:78-81.

Mevissen TET, Komander D (2017) Mechanisms of deubiquitinase specificity and regulation. Annu Rev Biochem 86:159-192.

Miesenböck G, De Angelis DA, Rothman JE (1998) Visualizing secretion and synaptic transmission with $\mathrm{pH}$-sensitive green fluorescent proteins. Nature 394:192-195.

O’Brien RJ, Kamboj S, Ehlers MD, Rosen KR, Fischbach GD, Huganir RL (1998) Activity-dependent modulation of synaptic AMPA receptor accumulation. Neuron 21:1067-1078.
Park EC, Glodowski DR, Rongo C (2009) The ubiquitin ligase RPM-1 and the p38 MAPK PMK-3 regulate AMPA receptor trafficking. PLoS One 4: e4284.

Park M, Penick EC, Edwards JG, Kauer JA, Ehlers MD (2004) Recycling endosomes supply AMPA receptors for LTP. Science 305:1972-1975.

Prekeris R, Klumperman J, Chen YA, Scheller RH (1998) Syntaxin 13 mediates cycling of plasma membrane proteins via tubulovesicular recycling endosomes. J Cell Biol 143:957-971.

Prekeris R, Foletti DL, Scheller RH (1999) Dynamics of tubulovesicular recycling endosomes in hippocampal neurons. J Neurosci 19:10324-10337.

Rodrigues EM, Scudder SL, Goo MS, Patrick GN (2016) A $\beta$-induced synaptic alterations require the E3 ubiquitin ligase Nedd4-1. J Neurosci 36:1590-1595.

Rongo C, Whitfield CW, Rodal A, Kim SK, Kaplan JM (1998) LIN-10 is a shared component of the polarized protein localization pathways in neurons and epithelia. Cell 94:751-759.

Schaefer H, Rongo C (2006) KEL-8 is a substrate receptor for CUL3-dependent ubiquitin ligase that regulates synaptic glutamate receptor turnover. Mol Biol Cell 17:1250-1260.

Schwarz LA, Hall BJ, Patrick GN (2010) Activity-dependent ubiquitination of GluA1 mediates a distinct AMPA receptor endocytosis and sorting pathway. J Neurosci 30:16718-16729.

Scudder SL, Goo MS, Cartier AE, Molteni A, Schwarz LA, Wright R, Patrick GN (2014) Synaptic strength is bidirectionally controlled by opposing activity-dependent regulation of Nedd4-1 and USP8. J Neurosci 34:1663716649.

Sowa ME, Bennett EJ, Gygi SP, Harper JW (2009) Defining the human deubiquitinating enzyme interaction landscape. Cell 138:389-403.

Turrigiano GG (2008) The self-tuning neuron: synaptic scaling of excitatory synapses. Cell 135:422-435.

Turrigiano GG, Leslie KR, Desai NS, Rutherford LC, Nelson SB (1998) Activity-dependent scaling of quantal amplitude in neocortical neurons. Nature 391:892-896.

van Roessel P, Elliott DA, Robinson IM, Prokop A, Brand AH (2004) Independent regulation of synaptic size and activity by the anaphase-promoting complex. Cell 119:707-718.

Wang R, Mellem JE, Jensen M, Brockie PJ, Walker CS, Hoerndli FJ, Hauth L, Madsen DM, Maricq AV (2012) The SOL-2/Neto auxiliary protein modulates the function of AMPA-subtype ionotropic glutamate receptors. Neuron 75:838-850.

Watson RT, Khan AH, Furukawa M, Hou JC, Li L, Kanzaki M, Okada S, Kandror KV, Pessin JE (2004) Entry of newly synthesized GLUT4 into the insulin-responsive storage compartment is GGA dependent. EMBO J 23:2059-2070.

Widagdo J, Chai YJ, Ridder MC, Chau YQ, Johnson RC, Sah P, Huganir RL, Anggono V (2015) Activity-dependent ubiquitination of GluA1 and GluA2 regulates AMPA receptor intracellular sorting and degradation. Cell Rep 10:783-795.

Widagdo J, Guntupalli S, Jang SE, Anggono V (2017) Regulation of AMPA receptor trafficking by protein ubiquitination. Front Mol Neurosci 10:347.

Yuasa-Kawada J, Kinoshita-Kawada M, Rao Y, Wu JY (2009a) Deubiquitinating enzyme USP33/VDU1 is required for Slit signaling in inhibiting breast cancer cell migration. Proc Natl Acad Sci USA 106:14530-14535.

Yuasa-Kawada J, Kinoshita-Kawada M, Wu G, Rao Y, Wu JY (2009b) Midline crossing and Slit responsiveness of commissural axons require USP33. Nat Neurosci 12:1087-1089.

Zhang X, Odom DT, Koo SH, Conkright MD, Canettieri G, Best J, Chen H, Jenner R, Herbolsheimer E, Jacobsen E, Kadam S, Ecker JR, Emerson B, Hogenesch JB, Unterman T, Young RA, Montminy M (2005) Genomewide analysis of cAMP-response element binding protein occupancy, phosphorylation, and target gene activation in human tissues. Proc Natl Acad Sci USA 102:4459-4464.

Zheng Y, Brockie PJ, Mellem JE, Madsen DM, Maricq AV (1999) Neuronal control of locomotion in C. elegans is modified by a dominant mutation in the GLR-1 ionotropic glutamate receptor. Neuron 24:347-361. 